\title{
A multi-wavelength classification method for polar stratospheric cloud types using infrared limb spectra
}

\author{
Reinhold Spang ${ }^{1}$, Lars Hoffmann ${ }^{2}$, Michael Höpfner ${ }^{3}$, Sabine Griessbach ${ }^{2}$, Rolf Müller ${ }^{1}$, Michael C. Pitts ${ }^{4}$, \\ Andrew M. W. Orr ${ }^{5}$, and Martin Riese ${ }^{1}$ \\ ${ }^{1}$ Forschungszentrum Jülich, Institut für Energie und Klimaforschung, Stratosphäre, IEK-7, Jülich, Germany \\ ${ }^{2}$ Forschungszentrum Jülich, Jülich Supercomputing Centre, JSC, Jülich, Germany \\ ${ }^{3}$ Karlsruhe Institut für Technologie, Institut für Meteorologie und Klimaforschung, Karlsruhe, Germany \\ ${ }^{4}$ NASA Langley Research Center, Hampton, VA, USA \\ ${ }^{5}$ British Antarctic Survey, Cambridge, UK \\ Correspondence to: Reinhold Spang (r.spang@fz-juelich.de)
}

Received: 20 January 2016 - Published in Atmos. Meas. Tech. Discuss.: 15 February 2016

Revised: 2 June 2016 - Accepted: 28 June 2016 - Published: 9 August 2016

\begin{abstract}
The Michelson Interferometer for Passive Atmospheric Sounding (MIPAS) instrument on board the ESA Envisat satellite operated from July 2002 until April 2012. The infrared limb emission measurements represent a unique dataset of daytime and night-time observations of polar stratospheric clouds (PSCs) up to both poles. Cloud detection sensitivity is comparable to space-borne lidars, and it is possible to classify different cloud types from the spectral measurements in different atmospheric windows regions.

Here we present a new infrared PSC classification scheme based on the combination of a well-established two-colour ratio method and multiple 2-D brightness temperature difference probability density functions. The method is a simple probabilistic classifier based on Bayes' theorem with a strong independence assumption. The method has been tested in conjunction with a database of radiative transfer model calculations of realistic PSC particle size distributions, geometries, and composition. The Bayesian classifier distinguishes between solid particles of ice and nitric acid trihydrate (NAT), as well as liquid droplets of super-cooled ternary solution (STS).

The classification results are compared to coincident measurements from the space-borne lidar Cloud-Aerosol Lidar with Orthogonal Polarization (CALIOP) instrument over the temporal overlap of both satellite missions (June 2006March 2012). Both datasets show a good agreement for the specific PSC classes, although the viewing geometries and the vertical and horizontal resolution are quite different. Dis-
\end{abstract}

crepancies are observed between the CALIOP and the MIPAS ice class. The Bayesian classifier for MIPAS identifies substantially more ice clouds in the Southern Hemisphere polar vortex than CALIOP. This disagreement is attributed in part to the difference in the sensitivity on mixed-type clouds. Ice seems to dominate the spectral behaviour in the limb infrared spectra and may cause an overestimation in ice occurrence compared to the real fraction of ice within the PSC area in the polar vortex.

The entire MIPAS measurement period was processed with the new classification approach. Examples like the detection of the Antarctic NAT belt during early winter, and its possible link to mountain wave events over the Antarctic Peninsula, which are observed by the Atmospheric Infrared Sounder (AIRS) instrument, highlight the importance of a climatology of 9 Southern Hemisphere and 10 Northern Hemisphere winters in total. The new dataset is valuable both for detailed process studies, and for comparisons with and improvements of the PSC parameterizations used in chemistry transport and climate models.

\section{Introduction}

Polar stratospheric clouds (PSCs) play an essential role in the depletion of stratospheric ozone (Solomon, 1999). Although they have been explored for more than 30 years, there are still many unanswered questions that limit our ability to predict 
the formation and surface area of different PSCs and, consequently, the prediction of future polar ozone loss rates in a changing climate system. With the continued implementation of the Montreal Protocol and its amendments and adjustments, a recovery of the ozone hole and the disappearance of the Antarctic ozone hole is projected to occur by the end of the century. However, there is a large uncertainty in estimates of the rate and timing of this recovery (Eyring et al., 2013; WMO, 2014). Accurate projections of the timing of recovery are critical, as they will further reshape Southern Hemisphere climate and weather (Polvani et al., 2011; Gerber and Son, 2014). These projections are necessary for deciding on climate change mitigation and adaptation policies.

The difficulty in making accurate predictions stems from a variety of problems of chemistry-climate models (CCMs), where one important problem is the poor representation of PSCs. CCMs used for assessments of stratospheric ozone loss (e.g. Eyring et al., 2013) often employ rather simple heterogeneous chemistry schemes. The simpler schemes are frequently based on nitric acid trihydrate (NAT), although it is known that heterogeneous chemistry on super-cooled ternary solution (STS) and on cold binary aerosol particles probably dominates polar chlorine activations (e.g. Solomon, 1999; Drdla and Müller, 2012). The models usually do not include comprehensive microphysical modules to describe the evolution of different types of PSCs over the winter. In addition, mesoscale temperature variations caused by gravity waves are crucial for the formation of PSCs under conditions close to temperature threshold conditions (e.g. Carslaw et al., 1998; Engel et al., 2013), but are missing from the current generation of CCMs (Orr et al., 2015).

PSCs are classified according to their composition into three types. This distinction is important as catalytic ozone destruction can be sensitive to PSC composition, and the formation of PSC types is extremely temperature sensitive. Super-cooled ternary solutions (STS) form by condensation of $\mathrm{HNO}_{3}$ and water vapour on stratospheric background sulfate aerosols. This occurs at temperatures $2-3 \mathrm{~K}$ below the existence temperature of solid nitric acid trihydrate (NAT) particles at $T_{\mathrm{NAT}} \sim 195 \mathrm{~K}$. The formation of NAT by homogeneous nucleation requires much lower temperatures. This is usually $3-4 \mathrm{~K}$ below the ice frost point $T_{\text {ice }}$ at $\sim 185 \mathrm{~K}$, where ice particles are formed (e.g. Peter and Grooß, 2012). The formation of STS droplets is well understood (e.g. Carslaw et al., 1995), but for NAT and ice particles, new formation mechanisms by heterogeneous nucleation on meteoric smoke well above $T_{\text {ice }}$ are under discussion (Hoyle et al., 2013; Engel et al., 2013; Grooß et al., 2014). These pathways for NAT and ice formation that are new so far are supported by the observation of an unusually large amount of refractory submicron aerosols of most likely meteoric origin in composition measurements of cloud condensation nuclei (Weigel et al., 2014). The widespread detection of PSC-containing NAT particles well above $T_{\mathrm{ICE}}$ in satellite observations in Northern Hemisphere winters without indication for orographic grav- ity waves is a distinctive indicator of a formation mechanism of NAT particles independent of pre-existing ice particles (Hoyle et al., 2013).

Progress on the evaluation of CCMs and chemical transport model (CTM) results is currently limited regarding PSC processes, as detailed long-term observations of PSCs are not available at present. Although solar occultation measurements have some capability for the discrimination of PSC types (e.g. Strawa et al., 2002; Zasetsky et al., 2007), they cannot be conducted in the polar night. The wintertime polar regions have been covered for several years by remote sensing satellite instruments which are not dependent on the sun as a light source. A 10-year archive (2002-2012) of measurements from the Michelson Interferometer for Passive Atmospheric Sounding (MIPAS) (Fischer et al., 2008) instrument on board ESA's Environment Satellite (Envisat) and ongoing measurements from the Cloud-Aerosol Lidar with Orthogonal Polarization (CALIOP) instrument from the NASA/CNES Cloud-Aerosol Lidar and Infrared Pathfinder Satellite Observations (CALIPSO) mission (Winker et al., 2009), which started in May 2006, are available.

This paper introduces a new classification method of the composition of PSCs based on infrared (IR) limb measurements and first applications of the MIPAS data. After the introduction, the paper will present details on instruments and datasets used in the analyses (Sect. 2), followed by a review of both formerly adopted and new classification methods (Sect. 3). Supported by radiative transfer calculations and the MIPAS measurements themselves, all methods are combined in a new Bayesian classifier (BC) for a comprehensive PSC composition classification for MIPAS. Section 4 presents some examples of the completely processed dataset, as well as a comparison with the CALIOP instrument.

\section{Instruments and datasets}

\subsection{MIPAS instrument on Envisat}

MIPAS on board the Envisat satellite measured limb infrared (IR) spectra in the wavelength range from 4 to $15 \mu \mathrm{m}$ (Fischer et al., 2008) from July 2002 to April 2012. The satellite operated on a sun synchronous orbit (inclination $98.4^{\circ}$ ) and allowed geographical coverage up to both poles due to additional poleward tilt of the primary mirror. The high spectral resolution of $0.025 \mathrm{~cm}^{-1}$ (HR: high-resolution mode) was reduced in 2004 to $0.0625 \mathrm{~cm}^{-1}$ (OR: optimized-resolution mode) due to technical problems with the interferometer (Raspollini et al., 2013). Consequently, the level $1 \mathrm{~b}$ radiance data from the measurement period July 2002 to March 2004 (Phase 1) were measured in the HR mode and from January 2005 to March 2012 (Phase 2) in the OR mode. Vertical and horizontal sampling for the nominal measurement modes are changed from Phase 1 to 2 . In Phase $1 \mathrm{a}$ constant $3 \mathrm{~km}$ grid was used up to a tangent height of $42 \mathrm{~km}$. In Phase 2 this 
changed to a latitude-dependent vertical step size of $1.5-$ $4.5 \mathrm{~km}$ (starting tangent altitudes ranging from 5 to $70 \mathrm{~km}$ at the poles to $12-77 \mathrm{~km}$ over the equator), with steps increasing with height from 1.5 to $4.5 \mathrm{~km}$. The horizontal sampling changed from $550 \mathrm{~km}$ to $420 \mathrm{~km}$. The vertical field of view (FOV) of MIPAS has a trapezoidal form with a base width of $4 \mathrm{~km}$ and a minimum width of $\sim 2.8 \mathrm{~km}$. The cross-track FOV is $30 \mathrm{~km}$.

\subsubsection{MIPAS cloud measurements}

In the following analyses we make use of the cloud detection results of the MIPclouds processor (Spang et al., 2012) Version 1.2.0, a prototype processor originally developed during an ESA study on fast cloud processor products for MIPASEnvisat (Spang et al., 2010a, b). Various detection methods are applied in the processor, such as the cloud index (CI) colour ratio approach (Spang et al., 2004) with constant and variable detection threshold values, as well as being variable in latitude and altitude (Spang et al., 2012; Sembhi et al., 2012). In addition, a retrieval approach with simplified assumptions for the radiative transfer in clouds (Hurley et al., 2011) is implemented. The retrieval copes with the difficulty of using a $3 \mathrm{~km}$ vertical FOV to determine a more realistic cloud top height $(\mathrm{CTH})$ inside the FOV. A step-like data processing approach of up to five methods was chosen for the MIPclouds processor to provide summary CTH information with the best possible detection sensitivity (Spang et al., 2012). We use this approach for the detection of the first cloud-affected spectrum in an altitude scan for starting with the PSC classification.

Figure 1 shows examples of PSC spectra measured by MIPAS and classified as ice, NAT, and STS. The spectral resolution is reduced to $1.2 \mathrm{~cm}^{-1}$ by applying a Gaussian instrument line shape with corresponding width. The resulting resolution is usually sufficient to highlight differences in the continuum-like emission of the different PSC types. Aerosol and cloud particles rarely produce narrow spectral features, and the strong spectral signature of NAT at $820 \mathrm{~cm}^{-1}$ is an exception (Spang and Remedios, 2003; Höpfner et al., 2006a).

The MIPAS detection sensitivity for clouds and aerosol is excellent. Due to the long limb path through the tangent height layer (i.e. $\sim 400 \mathrm{~km}$ for a $3 \mathrm{~km}$ vertical FOV at $20 \mathrm{~km}$ altitude) the instrument integrates all scattered and emitted radiation from cloud particles of a large volume of air spread along the line of sight. Based on radiative transfer modelling, Spang et al. (2015) estimated the detection sensitivity of an IR limb sounder with respect to the ice water content (IWC) for cirrus clouds in the lowermost stratosphere. These conditions are transferable to ice PSCs in the polar vortices. A cloud layer with $1 \mathrm{~km}$ horizontal extent should be detectable with IWC $>0.3 \mathrm{mg} \mathrm{m}^{-3}$ (and therefore a $100 \mathrm{~km}$ extent with IWC $>0.003 \mathrm{mg} \mathrm{m}^{-3}$ ), if the vertical and cross-track direction of the FOV is completely filled by the cloud. This rep-
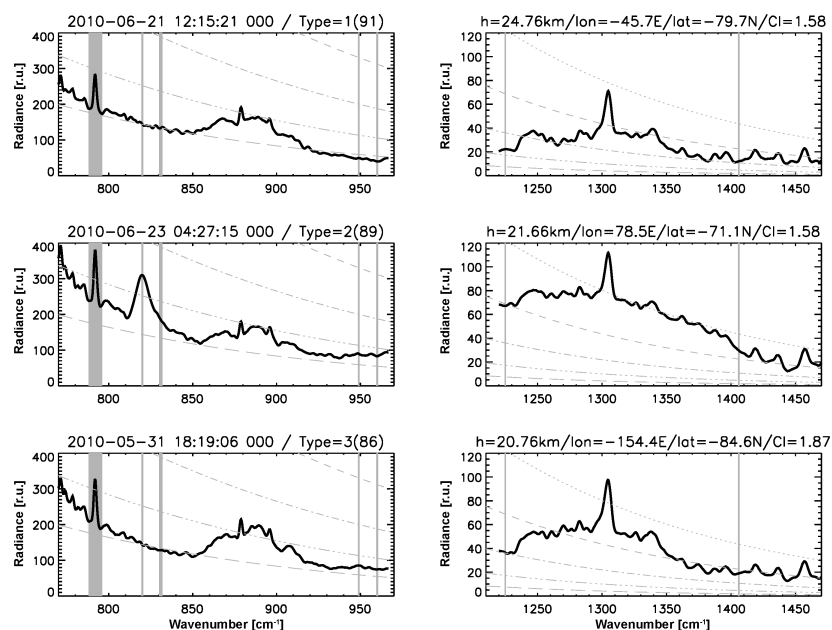

Figure 1. Examples of MIPAS radiance spectra in radiance units $\left(1 \mathrm{r} . \mathrm{u} .=1 \mathrm{nW} /\left(\mathrm{cm}^{2} \mathrm{srcm}^{-1}\right)\right)$ in the two main wavelength regions of interest. Spectra are classified into ice (top), NAT (middle), and STS (bottom) with high confidence in classification $\left(P_{\text {type }}=91 / 89 / 86 \%\right.$, see Sect. 3.3). Superimposed grey bars are the selected wavelength regions for the classification scheme. The spectral resolution is degraded to $\sim 1.2 \mathrm{~cm}^{-1}$ with a sampling of $0.35 \mathrm{~cm}^{-1}$. Planck functions for 140 to $180 \mathrm{~K}$ in $10 \mathrm{~K}$ steps are superimposed in grey dash/dotted curves.

resents an extremely high detection sensitivity, even better than most of the current tropospheric cloud products of the CALIOP lidar, for which most products typically are based on $5 \mathrm{~km}$ horizontal averaging (Avery et al., 2012).

An algorithm for PSC type classification was already part of the MIPclouds processor but showed various problems and spurious results in comparison with space-borne and groundbased measurements. Therefore, we developed a new more reliable classification scheme, partly based on methods already applied in the MIPclouds processor, and a new approach for the combination of several brightness temperature differences with these methods.

\subsection{Cloud scenario database}

As part of the MIPclouds study (Spang et al., 2012), a comprehensive cloud scenario database (CSDB) was compiled of modelled MIPAS radiance spectra in the presence of various cloud types and different atmospheric conditions. The database contains more than 70000 different cloud scenarios and more than 600000 spectra affected by PSCs (STS, NAT, and ice), cirrus, and liquid water clouds (Spang et al., 2008).

An optimized list of window regions was selected for the database with a total spectral range of $137 \mathrm{~cm}^{-1}$ : 782-841, 940-965, 1224-1235, 1246-1250, 1404-1412, 1929-1935, 1972-1985, 2001-2006, and $2140-2146 \mathrm{~cm}^{-1}$. The selection of the spectral windows was performed by calculating broadband Jacobians with respect to aerosol extinction at tangent levels with and without trace gas contribution. In a first-order 
Table 1. Summary of cloud scenario database parameter space.

\begin{tabular}{lll}
\hline PSC type & Volume densities $\left(\mu \mathrm{m}^{3} \mathrm{~cm}^{-3}\right)$ & Median radius $(\mu \mathrm{m})$ \\
\hline Ice & $10,50,100$ & $1.0,2.0,3.0,4.0,5.0,10.0$ \\
NAT & $0.1,0.5,1.0,5.0,10.0$ & $0.5,1.0,2.0,3.0,4.0,5.0$ \\
STS 02/48, 25/25, 48/02* & $0.1,0.5,1.0,5.0,10.0$ & $0.1,0.5,1.0$ \\
\hline Cloud top height $(\mathrm{km})$ & Cloud minimum bottom height $(\mathrm{km})$ & Cloud vertical extent $(\mathrm{km})$ \\
\hline 28.5-12.5 with $1 \mathrm{~km}$ spacing & $12-28$ & $0.5,1,2,4,8$ \\
\hline Modelled tangent heights: & $30 \mathrm{~km}$ down to $12 \mathrm{~km}$ with $1 \mathrm{~km} \mathrm{spacing}$ \\
\hline * Three weight percentages of $\mathrm{H}_{2} \mathrm{SO}$ and $\mathrm{HNO}_{3}$ are modelled (e.g. 02/48 means $2 \mathrm{wgt} \% \mathrm{H}_{2} \mathrm{SO}_{4}$ and $\left.48 \mathrm{wgt} \% \mathrm{HNO}_{3}\right)$.
\end{tabular}

approximation the quotient of these Jacobians is equal to the gas-transmission spectra at tangent altitude. The rationale of these simulations was to exclude regions of the spectrum with strong interference of trace gases and of already opaque intervals (Spang et al., 2008). A transmission limit of 0.9 was defined to select the wavelength regions. In addition, wavelength regions used in former studies (Spang et al., 2003, 2004, 2005a, b; Höpfner et al., 2006a, b) were taken into account.

The CSDB spectra were generated with the Karlsruhe Optimized and Precise Radiative transfer Algorithm (KOPRA) model (Stiller, 2000), which takes single scattering into account (Höpfner, 2004). Input parameters, such as effective radius, volume density, cloud type, and composition (e.g. three possible $\mathrm{H}_{2} \mathrm{SO}_{4} / \mathrm{HNO}_{3}$ compositions for STS: $02 / 48$, $25 / 25$, and $48 / 02 \%$ with $50 \% \mathrm{H}_{2} \mathrm{O}$ ) as well as cloud top and bottom height, were varied for the database. Table 1 presents a summary of the parameter space covered by the input parameters of the model runs, including the particle size distribution (PSD). Refractive indices for NAT by Biermann et al. (2000) and Höpfner et al. (2006a), for ice by Toon et al. (1994), and for STS by Biermann et al. (2000) were applied for the computation of the single scattering properties of spherical particles.

T-Matrix calculations with realistic bulk properties for cirrus clouds show that the scattering properties in the size range of PSC ice particles (effective radius $<10 \mu \mathrm{m}$ ) can be well approximated by Mie calculations and the particle shape is negligible (Baran, 2004; Yang et al., 2005). For NAT clouds Woiwode et al. (2014) found indications that aspherical particles might modify the spectral shape of the characteristic spectral NAT feature at $820 \mathrm{~cm}^{-1}$ in balloon-based IR measurements (Fig. 1). This very recent finding has been investigated in more detail in a parallel study (Woiwode et al., 2016) and is not considered below.

The radiative transfer calculations assume a homogeneous cloud layer, filling the tangent height layer with PSC particles. This simplified 1-D geometry is not generally applicable for a limb-scanning instrument. Under realistic atmospheric conditions, broken cloud fragments of various scales in front or behind the tangent point can be expected. Al- though these 2-D effects are not explicitly modelled in the database, various cloudy path lengths are already included by simulations of spectra below the minimum cloud bottom height (see Table 1). These geometries result in variable lengths of cloud segments along the line of sight. For example, a homogeneous cloud layer of $2 \mathrm{~km}$ thickness creates a maximum cloud path of $320 \mathrm{~km}$ exactly for a tangent height at the cloud bottom; but due to the spherical shape of the cloud layer, the line of sight of tangent heights below the cloud bottom crosses the homogeneous cloud layer above twice. For example, $3 \mathrm{~km}$ below the cloud bottom of a $2 \mathrm{~km}$ thick cloud layer, the effective cloud path shrinks to $2 \times 57 \mathrm{~km}=114 \mathrm{~km}$; for a cloud layer of only $0.5 \mathrm{~km}$ thickness a cloud path of $\sim 31 \mathrm{~km}$ remains.

Spang et al. (2012) showed that detection sensitivity and optical thickness in the limb strongly relate to the integrated volume density path (VDP) or the integrated area density path (ADP) along the line of sight. Subject to the particle median radius $r$, optical thickness is dominated by ADP for cloud types with larger particles $(r>5 \mu \mathrm{m}$, typically ice clouds) and VDP for small particles $(r<5 \mu \mathrm{m}$, like STS, NAT, and sulfate aerosols). The variable lengths of cloud segments in the CSDB result in a broad variability in ADP and VDP, which would occur in the real atmosphere for broken cloud segments along the line of sight. Finally, note the cloud index correlates well with VDP and ADP, depending on the cloud type. Spang et al. (2012) showed that CI is a useful proxy for the optical thickness of the cloud and that it is possible to estimate VDP or ADP from the CI measurements depending on the particle type.

\subsection{CALIOP instrument on CALIPSO}

The CALIOP instrument is a dual wavelength polarization sensitive lidar that provides high vertical resolution profiles of backscatter coefficients at 532 and $1064 \mathrm{~nm}$ (Winker et al., 2009). The CALIOP instrument is a part of the CALIPSO nadir viewing sun-synchronous satellite system inclined at $98^{\circ}$ at an altitude of $705 \mathrm{~km}$. The orbit geometry facilitates measurements up to latitudes of $82^{\circ} \mathrm{N} / \mathrm{S}$, compared with of $87^{\circ} \mathrm{S}$ to $89^{\circ} \mathrm{N}$ for MIPAS. 
In this study, we used lidar level 2 polar stratospheric cloud mask data, CAL_LID_L2_PSCMask-ProvV1-00 (CALIPSO, 2015). The CALIOP product comprises cloud and aerosol backscatter coefficient profiles at $5 \mathrm{~km}$ horizontal and $180 \mathrm{~m}$ vertical sampling size. The PSC detection and classification is so far limited to observations at night because higher levels of background light during daytime significantly reduce the signal-to-noise ratio. A successive horizontal averaging of $5,15,45$, or $135 \mathrm{~km}$ is applied to the data to improve the signal-to-noise ratio for the detection of optically thin clouds (typically necessary for STS and NAT clouds) (Pitts et al., 2009).

The second-generation CALIPSO PSC algorithm (Pitts et al., 2009) detects clouds using both the CALIOP $532 \mathrm{~nm}$ scattering ratio (the ratio of total to molecular backscatter) and the $532 \mathrm{~nm}$ perpendicular backscatter coefficient. The algorithm also includes a scheme for classifying PSCs by composition based on the measured CALIOP aerosol depolarization ratio and the inverse scattering ratio. Pitts et al. (2009) defined four PSC composition classes for the CALIPSO measurements: STS, water ice, and two classes (Mix 1 and Mix 2) of STS/NAT mixtures. Mix 1 denotes mixtures with very low NAT number densities (from about $3 \times$ $10^{-4} \mathrm{~cm}^{-3}$ - the inferred CALIOP NAT sensitivity threshold - to $1 \times 10^{-3} \mathrm{~cm}^{-3}$ ), while Mix 2 denotes mixtures with higher $\left(>10^{-3} \mathrm{~cm}^{-3}\right)$ NAT number densities.

The STS class may also include low number densities of NAT particles whose optical signature is masked by the more numerous liquid droplets of STS at cold temperatures (Pitts et al., 2013). High number densities of NAT particles, that are not masked by liquids, lie within the rest of the Mix 2 domain and are described as being Mix 2-enhanced (Mix 2enh), where a minority of ice clouds may also appear. Finally, the data show a subset of CALIPSO ice PSCs in the parameter space for intense mountain-wave-induced PSCs. Through their distinct optical signature in the scattering ratio and lidar colour ratio (the ratio of 1064 to $532 \mathrm{~nm}$ aerosol backscatter coefficients) this CALIPSO class is easily to separate from usual ice observations and is described as being wave ice. Consistent with mountain wave PSCs, this type is characterized by a high ice particle number density $(100 \%$ ice activation from the background aerosol) but relatively small particles (1-1.5 $\mu$ m radius, e.g. Pitts et al., 2009).

\subsection{AIRS instrument}

In this study, we use radiance measurements of the Atmospheric Infrared Sounder (AIRS) (Aumann et al., 2003; Chahine et al., 2006) to detect gravity waves in the polar lower stratosphere. AIRS is one of six instruments aboard the National Aeronautics and Space Administration's (NASA's) Aqua satellite. Aqua was launched in May 2002 and is the first satellite in NASA's "A-Train" constellation of satellites. Aqua operates in a nearly polar, sun-synchronous orbit (705 km altitude, $100 \mathrm{~min}$ period, $98.2^{\circ}$ inclination). AIRS measures about 2.9 million infrared nadir and sub-limb spectra per day. Spectral measurements cover the wavelength range from 3.74 to $15.4 \mu \mathrm{m}$ in three bands. Noise varies between 0.07 and $0.7 \mathrm{~K}$ at $250 \mathrm{~K}$ scene temperature. AIRS performs across-track scans, covering $1780 \mathrm{~km}$ distance on the ground and consisting of 90 footprints each. The footprint size of AIRS varies between $14 \times 14 \mathrm{~km}^{2}$ at nadir and $42 \times 21 \mathrm{~km}^{2}$ at the scan extremes. Due to its measurement geometry AIRS is capable of detecting gravity waves with short horizontal and long vertical wavelengths.

Here, gravity wave information is provided in terms of brightness temperature variances of the AIRS channels, covering the $15 \mu \mathrm{m}$ waveband of $\mathrm{CO}_{2}$, being most sensitive to atmospheric temperatures at about $15-40 \mathrm{~km}$ altitude, with a maximum in the weighting function around $20-30 \mathrm{~km}$. Spectral averaging and corrections to reduce noise and the detrending procedure for removing background signals due to large-scale temperature gradients or planetary waves follow the approach of Hoffmann et al. $(2013,2014)$.

\section{PSC type classification methods for IR limb sounding}

All classification methods presented below are based on characteristic spectral differences in the absorption and scattering efficiency of the three types of PSCs (STS, NAT, and ice) known to occur in the polar stratosphere (Peter and Grooß, 2012). At this point we should clarify that in the following, the terms "PSC type" and "mixed-type" are not related to the lidar-based classification of PSC observations of Type 1a, 1b, and 2 frequently used in the literature (e.g. Achtert and Tesche, 2014, and references therein). Here, the word "type" represents the three different types of particle composition, namely STS, NAT, and ice, which can affect and may dominate the spectral characteristics of a single measured infrared spectrum. The refractive indices of the particle type together with its size distribution are the key input parameters for the computation of the optical properties by the Mie theory (Mie, 1908), i.e. absorption and scattering efficiency and the scattering phase function. The wavelength dependence of the real $\left(n_{\mathrm{r}}\right)$ and imaginary $\left(n_{\mathrm{i}}\right)$ part of the complex refractive index $\left(r_{\mathrm{i}}\right)$ for ice, NAT, and STS is presented in Fig. 2, where $n_{\mathrm{i}}$ is responsible for the absorption and $n_{\mathrm{r}}$ for the scattering characteristic of the particle type. Obviously, gradients and value of $r_{\mathrm{i}}$ can be very different depending on the part of the spectral region and the particle type. The $r_{\mathrm{i}}$ values based on laboratory data are used in the radiative transfer calculations for the CSDB (Sect. 2.2). All wavelength regions of the final multi-wavelength classification method are superimposed in Fig. 2.

\subsection{Two-colour ratio method}

Originally, Spang and Remedios (2003) introduced the twocolour ratio method (2CR) for the classification of PSC spec- 

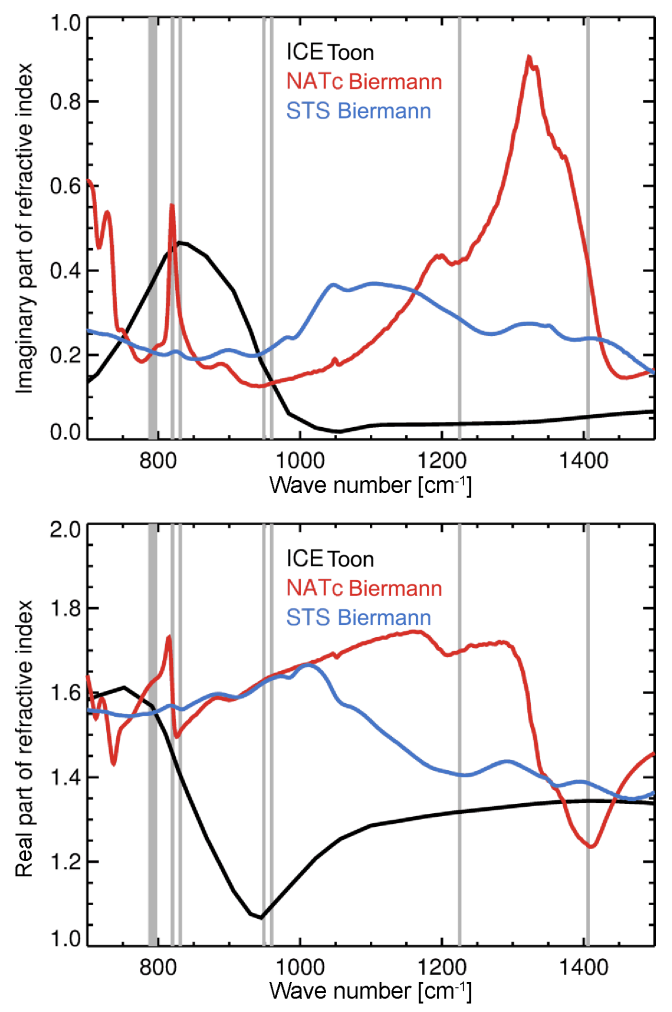

Figure 2. Refractive indices (imaginary part at top and real part at lower panel) of ice clouds (Toon et al., 1994), coated NAT (Biermann et al., 2000; Höpfner et al., 2006a), and STS with weight percentages of composition of $17 \mathrm{wgt} \% \mathrm{HNO}_{3}$ and $25 \mathrm{wgt} \% \mathrm{H}_{2} \mathrm{SO}_{4}$ (Biermann et al., 2000). The wavelength regions applied in the classification scheme are superimposed in grey.

tra based on Cryogenic Infrared Spectrometers and Telescopes for the Atmosphere (CRISTA) observations (Offermann et al., 1999; Grossmann et al., 2002). Höpfner et al. (2006a) further developed the method and compared the results with the CALIPSO PSC classification scheme (Höpfner et al., 2009). The 2CR method includes one colour ratio sensitive to the optical thickness and extinction of the cloud $\left(\mathrm{CR}_{1}=I_{11}\left(788.2-796.2 \mathrm{~cm}^{-1}\right) / I_{12}\right.$ $\left.\left(832.0-834.4 \mathrm{~cm}^{-1}\right)\right)$, which was originally introduced as the cloud index (CI) for the detection of cloudy and noncloudy spectra in the CRISTA and MIPAS radiance datasets (e.g. Spang et al., 2005). A second colour ratio is sensitive to a spectral feature attributed to the emissions of small NAT particles $\left(\mathrm{CR}_{2}=I_{21}\left(819.0-821.0 \mathrm{~cm}^{-1}\right) / I_{22}(788.2-\right.$ $796.2 \mathrm{~cm}^{-1}$ )) (Höpfner et al., 2006a), referred to in the following as the NAT index (NI). The method traces back to a study of the first observations of a NAT spectral signature at $820 \mathrm{~cm}^{-1}$ from space during the CRISTA-2 mission (Spang and Remedios, 2003). There is also a small but strong feature at this wave number range (Fig. 2), caused by the $\nu_{2}$ band emissions of $\mathrm{NO}_{3}^{-}$(Höpfner et al., 2002). In previous laboratory measurements of NAT refractive indices, the feature was not pronounced enough to reproduce the atmospheric infrared limb observations of the signature; but in a reanalysis of the Biermann et al. (2000) NAT film measurements in conjunction with radiative transfer model calculation, Höpfner et al. (2006a) showed the unambiguous assignment that NAT particles with $r<3 \mu \mathrm{m}$ produce the spectral signature in the measured spectra.

Figure 3 presents examples of the probability density distribution function (PDF) of CI vs. NI for MIPAS in the months May, June, August, and September for the Southern Hemisphere winter 2007. In the PDFs, additional separating lines (dash-dotted) are superimposed for regions in the parameter space where specific PSC types are dominating the distribution. The separating lines are retrieved from radiative transfer calculations for varying particle size distributions and composition (Höpfner et al., 2006a). They split the parameter space into one region above the curve, where events are definitely attributed to NAT with a particle median radius $r<3 \mu \mathrm{m}$. Larger particles of NAT and ice appear in the parameter space below the curve and are difficult to separate from STS, which are usually found in the area below the curve for $\mathrm{CI}>1.3$. Finally, spectra for ice are observed below the curve, but for $\mathrm{CI}<1.3$. Typical water vapour mixing ratios (2-6 ppm) and temperatures below $190 \mathrm{~K}$ in the polar winter stratosphere suggest the formation of ice clouds with volume densities of optically thick conditions in the limb direction. For example, a homogeneous ice water content of $1 \mathrm{ppmv}$ over the entire domain of the tangent height layer will produce an extinction of around $3 \times 10^{-2} \mathrm{~km}^{-1}$, which is equivalent to optically thick conditions in the limb direction (Spang et al., 2008) and values of $\mathrm{CI}<\sim 1.2$ (see also Fig. 1). The effective cloudy limb path is a crucial parameter for the observed optical thickness. The CI method is mainly sensitive to the limb-integrated volume density or area density path along the line of sight (Spang et al., 2012, 2015), and therefore small broken clouds fragments with high optical thickness along the line of sight can be detected with a moderate $\mathrm{CI}$ value. This is also the case for an optical thinner but horizontally extended cloud layers.

For the CSDB we used an extended parameter space (e.g. smaller volume densities for ice and larger for NAT and STS, see Sect. 2.2). This is because optically thinner ice clouds can form in the polar stratosphere or at least can remain after the sedimentation of larger particles. In addition, the potentially available $\mathrm{HNO}_{3}$ allows the formation of relatively high volume densities with $\mathrm{CI}<1.3$ NAT and STS clouds. Finally, the CSDB shows that the region attributed to ice can also include large NAT particles $(r>3 \mu \mathrm{m})$ or STS with large volume densities and corresponding large optical thickness. On the other hand, the STS $S_{\text {mix }}$ region can include optically thin ice clouds. The formation of relatively large volume densities for NAT and STS is conceivable for conditions with high $\mathrm{HNO}_{3}$ gas-phase values like for early winter with no denitrification or late winter in regions where re-nitrification takes place. 

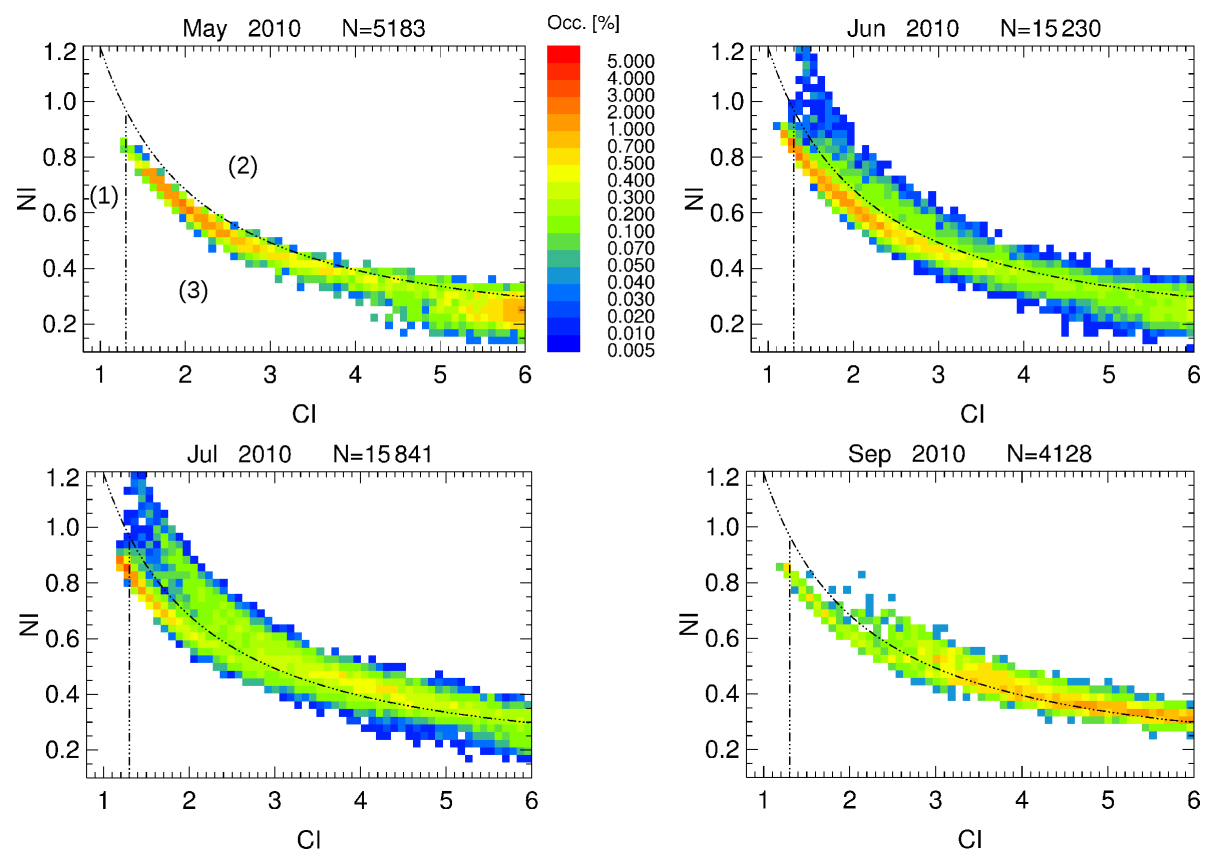

Figure 3. Probability density functions for cloud index (CI) vs. NAT index (NI) for Southern Hemisphere 2010 winter for the months May, June, August, and September (May 2007-September 2007) derived from MIPAS measurements in the altitude range 16-30 km. The percentage of occurrence with respect to the number of observations $\left(N_{\mathrm{obs}}\right)$ in $\mathrm{SH}$ polar cap region south of $55^{\circ} \mathrm{S}$ are colour-coded. The cloud type regions and separating lines are superimposed: region (1) for ice with overlap of high volume density STS (acronym ICE_STS H06; $_{3}$ see also Table 2), region (2) for small NAT (sNAT3 ${ }_{\mathrm{H} 06}$ ) particles, and region (3) for STS particles with overlap of large NAT particles (STS_lNAT ${ }_{\text {H06 }}$ ) based on Höpfner et al. (2006a).

Table 2. Predefined PSC type probability matrix for the Bayesian classifier (V1.2.8).

\begin{tabular}{|c|c|c|c|c|c|c|}
\hline $\begin{array}{l}\text { Region } \\
\text { index }\end{array}$ & Classifier & $\begin{array}{l}p_{\mathrm{ICE}} \\
(\%)\end{array}$ & $\begin{array}{l}p_{\mathrm{NAT}} \\
(\%)\end{array}$ & $\begin{array}{l}p_{\text {STS }} \\
(\%)\end{array}$ & $\begin{array}{l}\text { Acronym* } \\
\text { region }\end{array}$ & $\begin{array}{l}\text { Figure } \\
\text { ref. }\end{array}$ \\
\hline 1 & CI-NI (2CR) & 10 & 60 & 30 & $s \mathrm{NAT}^{\mathrm{H} 06}$ & Fig. 3 \\
\hline 2 & & 20 & 25 & 55 & STS_lNAT ${ }_{\mathrm{H} 06}$ & \\
\hline 3 & & 50 & 20 & 30 & ICE_STS & \\
\hline 4 & CI-BTD & 70 & 20 & 10 & $\mathrm{ICE}_{\mathrm{ci}}$ & Fig. 5 \\
\hline 5 & & 60 & 30 & 20 & $\mathrm{ICE}_{\mathrm{ci} i} s \mathrm{NAT} 1$ & \\
\hline 6 & & 10 & 40 & 50 & $\mathrm{STS}_{\mathrm{ci}-l \mathrm{NAT} 2}$ & \\
\hline 7 & $\mathrm{BTD}_{820-831}$ & 60 & 10 & 30 & $s$ ICE5 & Fig. 6 \\
\hline 8 & vs. $\mathrm{BTD}_{1406-960}$ & 30 & 30 & 40 & lICE5_STS_lNAT3 & \\
\hline 9 & & 10 & 50 & 40 & $m \mathrm{NAT}$ & \\
\hline 10 & & 10 & 60 & 30 & $s$ NAT2 & \\
\hline 11 & $\mathrm{BTD}_{831-1225}$ & 60 & 30 & 10 & ICE & Fig. 7 \\
\hline 12 & vs. BTD $960-1225$ & 50 & 30 & 20 & $l \mathrm{ICE} \_s \mathrm{NAT}$ & \\
\hline 13 & & 10 & 40 & 50 & STS_lNAT & \\
\hline
\end{tabular}

* Indices in the acronyms are indicated as follows: "H06", the separation lines based on Höpfner et al. (2006a); italic letters $l, m$ and $s$ large-, medium-, and small-sized particles, respectively; the numbers attached to the PSC type represent a rough guideline to the thresholds of the median radius $R_{\text {med }}$ and base on the input parameter of CSDB particles size distributions of Table 1 .

Although these uncertainties restrict the classification capability, the 2CR method turned out to be an extremely valuable tool for the analysis of PSC type distribution over Antarctic and Arctic winter periods (Spang et al., 2005a, b; Höpfner et al., 2006b; Eckermann et al., 2009). The ap- plication of this method to MIPAS allowed the detection of an Antarctic stratospheric belt of NAT PSCs caused by mountain waves for the first time (Höpfner et al., 2006b). In addition, the method showed a reasonable agreement with 
ground-based and space-borne lidar measurements of PSC composition (Höpfner et al., 2009).

The PDFs in Fig. 3 show a characteristic and expected development over the winter. In early winter (May), STS or $\mathrm{STS}_{\text {mix }}$ clouds dominate the PDF, which is in line with temperatures at this time of the year. Synoptic temperatures in May are usually not cold enough to form ice $\left(T_{\mathrm{ICE}} \sim 187 \mathrm{~K}\right)$. Consequently, little indication for NAT particles is found, whereas in June, NAT events become more prominent. Temperature perturbations induced by mountain waves over the Antarctic Peninsula may cause a NAT-cloud seeding, which can affect the entire outer vortex region (Höpfner et al., 2006b; Eckermann et al., 2009). In August, temperatures are cold enough to form ice and NAT in large areas of the polar vortex, causing two maxima in the distribution. In September the synoptic temperatures rise significantly above the $T_{\text {ice }}$ threshold, where NAT is also more difficult to form and the distribution show less pronounced maxima. The method still suggests a strong probability for NAT clouds, which is disputable under the assumption that NAT is only formed for $T<T_{\text {ice. However, the observations are in line with recent }}$ results of Engel et al. (2013) and Hoyle et al. (2013), showing evidence for a process of heterogeneous NAT nucleation at $T>T_{\text {ice }}$ for Arctic PSC events.

\subsection{Brightness temperature difference methods}

The 2CR method introduced above includes three different wavelength regions within the $790-833 \mathrm{~cm}^{-1}$ range and does not make use of the rather broad spectral ranges covered by MIPAS. Figure 2 indicates further differences in refractive indices of different PSC types. Therefore, we investigated the benefit of adding further spectral regions in detail.

\subsubsection{Combined CR-BTD classification}

Analyses of brightness temperature differences (BTDs) are frequently applied to nadir sounders for the differentiation of tropospheric aerosol, liquid, and ice water clouds (e.g. Li et al., 2003; Clarisse et al., 2013). Usually the methods use the characteristic strong gradient in absorption and scattering efficiency between $\sim 800$ and $\sim 950 \mathrm{~cm}^{-1}$ (see also Fig. 2) for the differentiation of ice from other aerosol types. This characteristic gradient has been exploited in several recent studies using MIPAS observations (Spang et al., 2012; Grainger et al., 2013; Griessbach et al., 2014).

A first attempt to apply this wavelength dependence to limb measurements was realized in an ESA study for the development of a fast cloud parameter processor for MIPAS (Spang et al., 2010, 2012). Figure 4 shows a CR-BTD combination with the colour ratio CI vs. $\mathrm{BTD}_{833-849}=\mathrm{BT}\left(833 \mathrm{~cm}^{-1}\right)-\mathrm{BT}\left(949 \mathrm{~cm}^{-1}\right)$ of the $\bmod -$ elled CSDB spectra. A significant separation is observable between ice and STS. However, NAT and STS have a relative similar distribution. Large NAT particles lose their character-
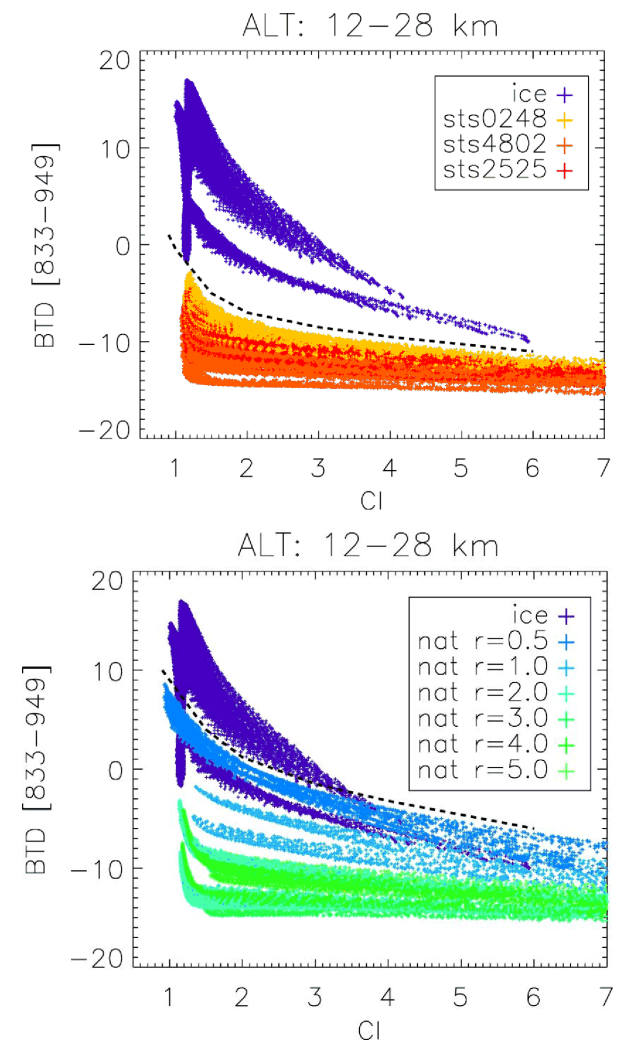

Figure 4. Modelled cloud index (CI) vs. brightness temperature difference between 833 and $949 \mathrm{~cm}^{-1}$ for all PSC scenarios of the CSDB. Top: for ice compared to three STS compositions (colourcoded; see also Table 1), and bottom: ice compared to NAT (colourcoded NAT radii). Superimposed dashed lines separate ice events from the corresponding second PSC composition in the figure, STS (top) and NAT (bottom), respectively.

istic wavelength dependence in the radiance emissions and an explicit differentiation becomes difficult in a large overlap region for NAT and STS.

Examples of two PDFs of the MIPAS CR-BTD distribution are presented in Fig. 5 for Northern Hemisphere (NH) winter 2010/11, a record winter for Arctic ozone destruction (e.g. Manney et al., 2011). During this outstanding NH winter, CALIOP observed unusual high occurrence rates for ice clouds. Typically, for most of the NH winter distributions (see examples) and for Southern Hemisphere (SH) early winters (May, not shown) there is no activity observed in the area attributed to ice and only occasionally in the ice-NAT area. We take into account that the spectra influenced by cloud emission show CI smaller than 5-6 depending on altitude (Spang et al., 2005a; Höpfner et al., 2006a), and typical detection thresholds are usually even a bit smaller (4.0-4.5). Starting in February 2011 (Fig. 5, bottom) for a phase of 46 weeks, synoptic cold temperatures in the range well below $T_{\text {NAT }}$ and close to $T_{\text {ice }}$ are observed (Arnone et al., 2012; Manney et al., 2011). This fits to the MIPAS observations for 

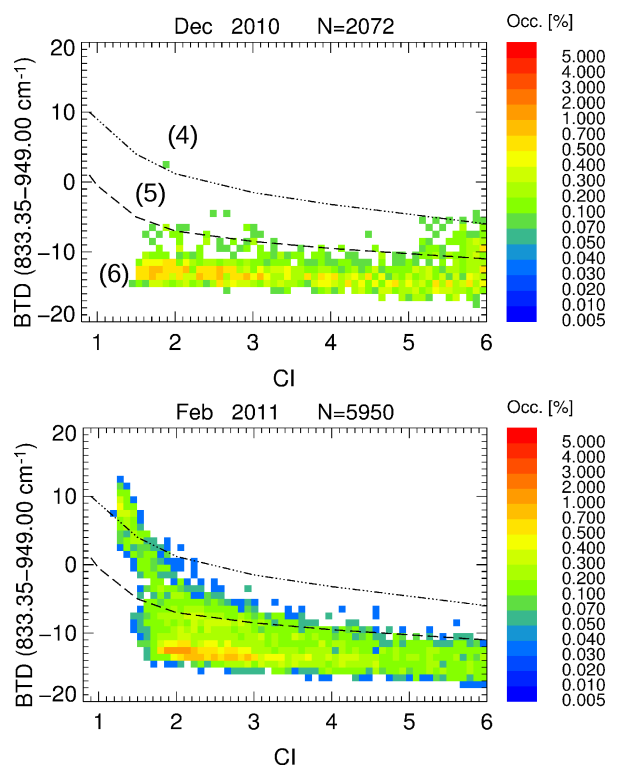

Figure 5. MIPAS 2-D PDF distribution of cloud index (CI) vs. BTD $833-949$ for the December 2010 (top) and February 2011 (bottom) measurement periods in the NH Arctic polar cap region (north of $55^{\circ} \mathrm{N}$ ) in the altitude range $16-30 \mathrm{~km}$. Superimposed separation functions (dashed-dotted and dashed lines) are retrieved from the CSDB in Fig. 4 and are highlighting region (4) for ice (acronym $\mathrm{ICE}_{\mathrm{ci}}$ in Table 2), region (5) for ice with overlap of small NAT particle emissions ( ICE $_{\mathrm{ci}} s \mathrm{NAT} 1$ ), and region (6) for the overlap of STS with large NAT $\left(\right.$ STS $_{\text {ci_l }}$ NAT2) particles (bottom region).

February with a large number of cloud events with significant ice signatures typical for SH midwinter conditions.

\subsubsection{2-D BTD classification methods}

To further improve the PSC classification, tests with various 2-D BTD PDFs and scatter diagrams of the MIPAS measurements and the CSDB were performed with major focus on the atmospheric window regions so far not considered in the two classification methods discussed above. Randomly chosen mean radiance pairs, spectrally integrated over $1 \mathrm{~cm}^{-1}$ intervals, were considered in this test. The main intention was to find some additional information to better discriminate STS and NAT clouds. This is non-trivial because the optical properties become very similar between both types if the NAT particles have a large radius, like the so-called NAT rocks (Fahey et al., 2001). Taylor et al. (1994) exploited the different gradients in the extinction spectrum of sulfate and NAT in the 1400 and $1200 \mathrm{~cm}^{-1}$ region for aerosol and PSC measurements of the Improved Stratospheric and Mesospheric Sounder (ISAMS) on the Upper Atmospheric Research Satellite (UARS).

Finally, two additional wavelength regions at 1225 and $1406 \mathrm{~cm}^{-1}$ were selected to add most complementary information for the classification of PSC. The $1406 \mathrm{~cm}^{-1}$ radiances add some radius sensitivity in the NAT/STS differenti-
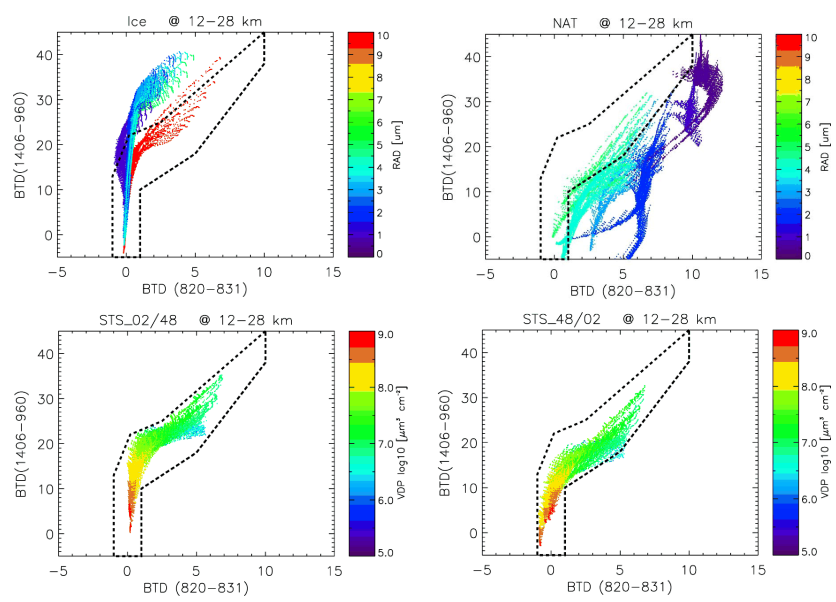

Figure 6. Scatter diagrams of $\mathrm{BTD}_{820-831}$ vs. $\mathrm{BTD}_{1406-960}$ distribution for the CSDB spectra of Ice (top, left) and NAT (top, right) with colour-coded radius dependence and two STS compositions (bottom) with colour-coded volume density path (VDP) dependence. The axes ranges are restricted to the BTD ranges typically observed for MIPAS (see next figure). Only data with CI $<6$ are presented. The superimposed polygon (dashed line) marks the area where spectra of modelled STS composition occur in the full set of the CSDB spectra.

ation in the $\mathrm{BTD}_{820-831}$ vs. $\mathrm{BTD}_{1406-960}$ distribution. The first BTD has its main contrast with respect to enhanced emissions at $820 \mathrm{~cm}^{-1}$ caused by small NAT particles (cf. Fig. 6), but the ice distribution also shows some radius sensitivity for BTD $820-831$. STS clouds tend to appear in regions separated from NAT and highlighted by the dashed polygon. Only a part of the large radius NAT clouds ( 3 and $5 \mu \mathrm{m}$ ) are able to overlap the area attributed to STS. BTD $1406-960$ seems to improve the separation of the medium NAT particles $(1-3 \mu \mathrm{m})$ from STS. In addition, it is sensitive to the optical thickness of the cloud. The two BTDs tend to shrink close to zero for optically thicker conditions (see colour-coded volume density path for STS). However, ice clouds with high optical thickness show a significant overlap exactly in this region, even for small and large mean radii (Fig. 6, top left). Consequently, this single classifier is not sufficient for a definite separation of ice and STS, but can still provide additional information for a combined classification approach.

Figure 7 presents PDF distributions of MIPAS measurements for the same BTDs shown in Fig. 6 for $\mathrm{NH}$ and $\mathrm{SH}$ winter months. Over the SH winter 2010, the distributions indicate that STS particles dominate the early winter (May). For June the maximum shifts to the left shoulder of region (8), where STS but also optically thicker ice clouds would appear and is expected due to colder synoptic temperatures, while in July the more pronounced NAT formation results in a bimodal distribution with a second maximum in region (10). The bimodality stays until late winter (September) and is typical for all SH late winter conditions, 

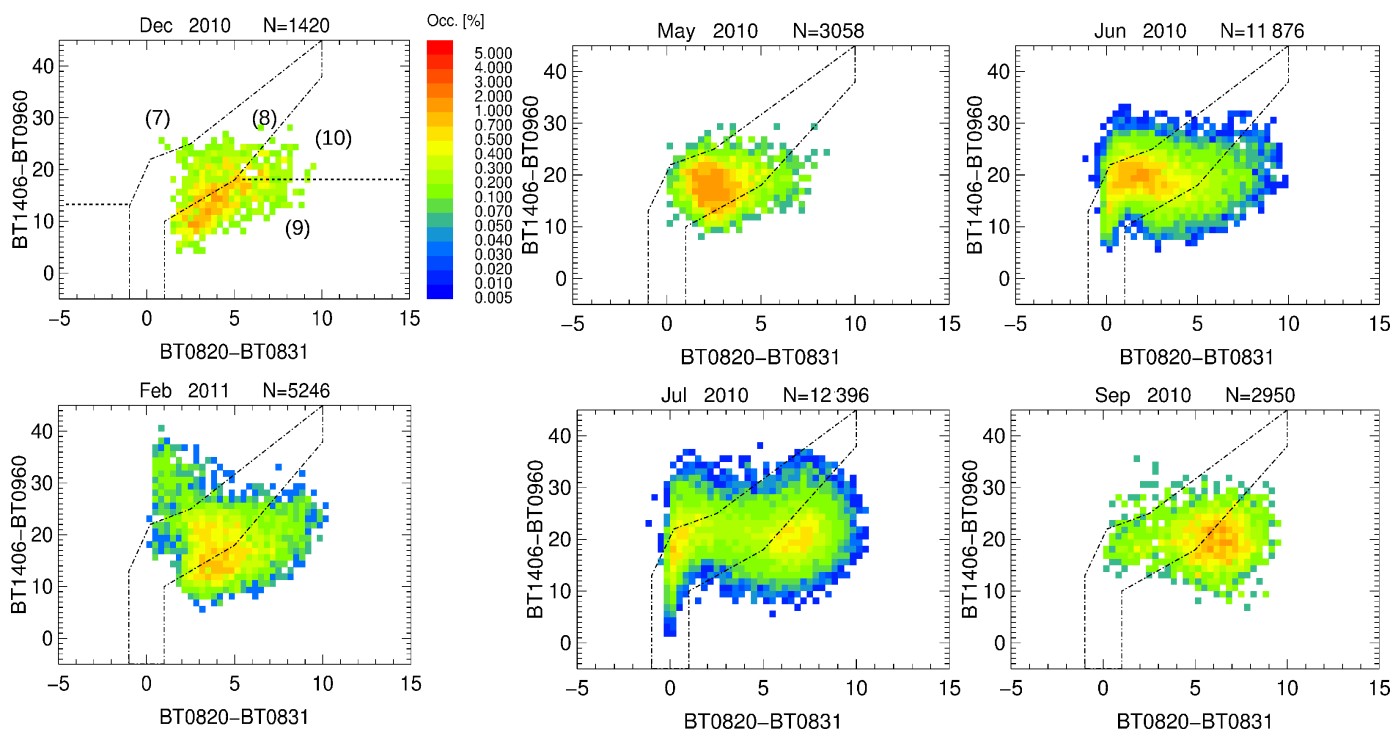

Figure 7. Monthly PDFs of $\mathrm{BTD}_{820-831}$ vs. BTD $1406-960$ from MIPAS for NH December 2010 and February 2011 (left column, like in Fig. 3) and SH May, June, July and September 2010 (middle and right column, like in Fig. 3) in the altitude range 16-30 km. The colour code represents the relative occurrence in percent with respect to the total number of cloudy spectra $\left(N_{\mathrm{obs}}\right)$ with $\mathrm{CI}<5$ for each individual spectrum observed north and south of $55^{\circ} \mathrm{N} / \mathrm{S}$ respectively. The dashed dotted polygon highlights regions (7)-(10) for various PSC compositions retrieved from the CSDB: region (8) represents the area of STS occurrence (Fig. 6) but may overlap with large ice and large NAT particles and is also indicated with the acronym $l$ ICE5_STS_lNAT3 in Table 2. The two horizontal lines (dashed) in this diagram create three additional areas where in region (7) small ice ( $s$ ICE5) dominates, in region $(9)$ medium-sized NAT particles $(m$ NAT) dominate, and in region (10) small NAT particles ( $s$ NAT2) dominate the distribution.
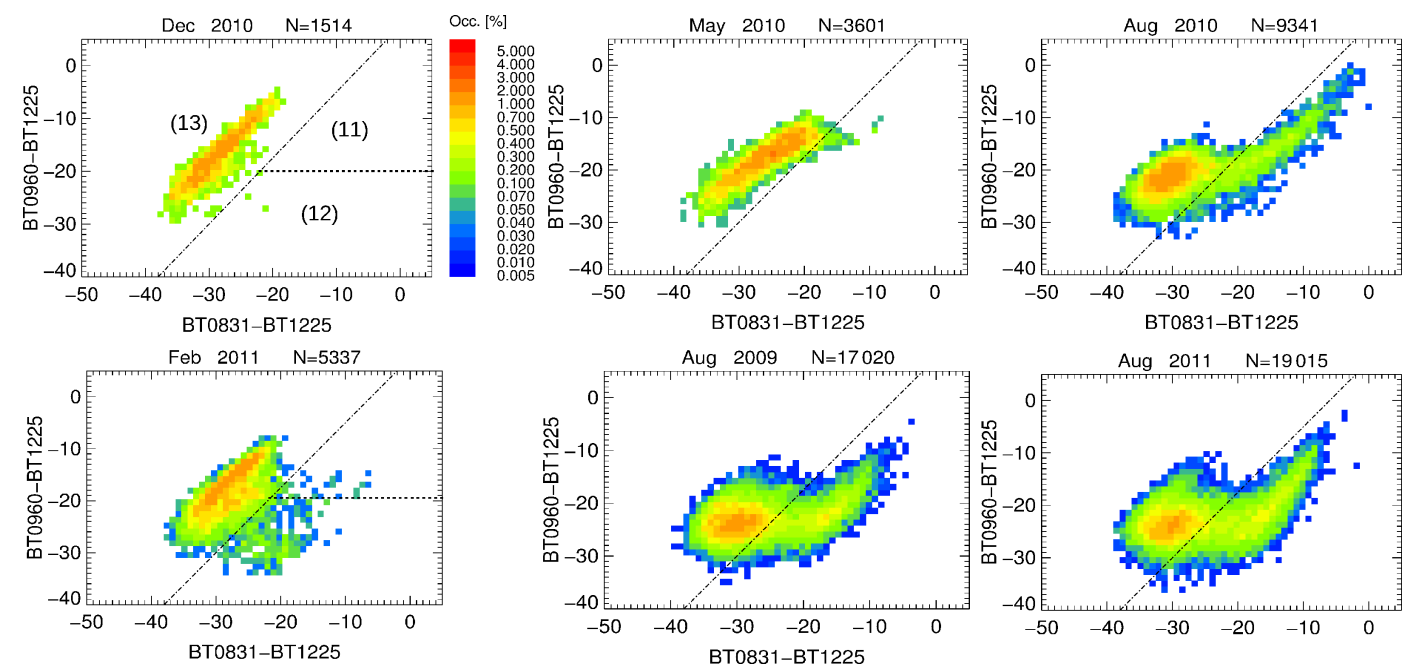

Figure 8. Monthly PDFs of $\mathrm{BTD}_{831-1225}$ vs. BTD $960-1225$ are presented for $\mathrm{NH}$ and $\mathrm{SH}$ conditions north and south of $55^{\circ} \mathrm{N} / \mathrm{S}$, respectively. NH December 2010, February 2011 (left column, corresponding to Figs. 3 and 5), SH May 2010, August 2010, as well as August 2009, and 2011 (middle and right column) for all cloudy observations $(\mathrm{CI}<5)$ in the altitude range $16-30 \mathrm{~km}$. Areas for PSC classification regions $(11)$, (12), and (13) are highlighted by dashed lines in the first figure (top left), where (11) is dominated by ice, (12) by large ice and small NAT ( $l$ ICE_sNAT; see also Table 2), and (13) by STS and large NAT particles (STS_lNAT).

like the other MIPAS SH winter observations suggest. Due to the much shorter PSC season in the NH and consequently less frequent formation of ice, the PDF looks very different. The small ice particle appearance in region (7) and events in the upper left corner of region (8) are only observed in a few years of the monthly PDFs for the NH. However, 3 out of 10 very cold winters in the MIPAS measurement period show an extension of the distribution into region (7). Finally, the incorporation of the wavenumber region at $1225 \mathrm{~cm}^{-1}$ improved the discrimination of ice from NAT and STS using a 
BTD $_{831-1225}$ vs. BTD $960-1225$ scatter diagram of the CSDB distribution (not shown).

Figure 8 shows examples for the MIPAS 2010/11 measurements, again for NH (January 2010 and February 2011) and four for SH conditions (again May 2010, but now August 2010 in comparison to August 2009, and 2011). The CSDB showed a distinctive sensitivity for a better ice discrimination and some radius sensitivity for the new BTD combination. Only ice particles of large radius $(10 \mu \mathrm{m})$ and low ADP (Sect. 2.1.1) can appear close and below the borderline to the region attributed to STS and NAT in region (13). All other modelled ice spectra appear below the borderline and ice clouds with small radii, but high ADP (high extinction/optical thickness) cause the elongation in direction toward the top right corner of the distribution. Obviously, the ice microphysical parameters seem to change for different Antarctic August conditions (Fig. 8).

\subsection{Bayes classification approach}

The BTD and CR combinations presented above clearly provide additional information on microphysical parameters (e.g. radius, ADP/VDP) as well as on the specific cloud type. However, discrimination with borderlines in 2-D PDF distribution, as presented earlier, is often difficult because a reliable classification of events close to the threshold curves is not possible. In the following, we apply a simple probabilistic classifier based on applying Bayes' theorem with strong (naive) independence assumptions to the four BTD and CR methods described in Sects. 3.1 and 3.2. Methods for the combination of multiple classification schemes like in the sections above, sometimes entitled "multiple discriminant analyses" in the literature, are described, for example, in Wilks (2005, chap. 13.3, with chap. 13.3.3 on the Bayesian approach). Bayesian methods have been applied in recent and former studies on cloud properties, mainly for algorithms on cloud detection for nadir sounders (Hollstein et al., 2015 and references therein). Here, we apply the method to combine the information content of the individual classification methods (classifier) into a single estimate of the most probable PSC type dominating the measured IR spectrum, referred to in the following as the Bayesian classifier (BC).

The four individual classifiers include 13 different classification areas in total, as presented in Figs. 3, 5, 7, and 8. For each of the 13 areas a probability of classification for ice, NAT, and STS $p_{i, j}$ is presented in the matrix of Table 2, where the indices $i=1, \ldots, 13$ and $j=1,2,3$ refer to the classification area and cloud type, respectively. In an area in the parameter space where the modelled spectra of the CSDB suggest that only one PSC particle type occurs (little or no overlap with other types) we attributed a large probability for the specific type and only small probabilities for the other two types (e.g. region 4 in Fig. 5 for ice). For areas with significant overlap, the probabilities are defined of similar size (equal probabilities of all $n=3$ cloud types would result in $p_{i, j}=33 \%$ ). A cloudy spectrum appears in one area of the classification diagrams of the four classifier $(m=4)$. The $\operatorname{index}(k)$ (Table 2) with $k=1 \ldots m$ will select always only one area per classifier (4 out of 13 in total), and represents exactly where the spectrum appears in the parameter space. Finally, we define a normalized product probability for each potential cloud type:

$P_{j}=\prod_{k=1}^{m} p_{\text {index }(k), j} / \sum_{j=1}^{n}\left(\prod_{k=1}^{m} p_{\text {index }(k), j}\right)$.

This approach attributes a probability for each of the three PSC types to each cloud spectrum. The maximum of the normalized probability indicates the most likely PSC type. For a more distinctive classification approach we introduced a threshold of $P_{j}>50 \%$ for a significant confidence that PSC type $j$ dominates the measured cloud spectrum. In addition, if two types have a probability between 40 and $50 \%$, this may give indication of a mixed-type cloud. This circumstance appears nearly exclusively in the data analysis for the combination NAT with STS (this mixture is named NAT_STS in the following), which most likely occurs in the analysis due to the difficulties of differentiating large NAT from STS particle clouds in the MIPAS measurements. It is also reasonable that mixed-type clouds or "sandwich" structures of both types (Shibata et al., 1997) in the FOV of the instrument generate this kind of event. Ground-based (e.g. Achtert and Tesche, 2014) and space-borne (e.g. Pitts et al., 2011) lidar measurements with better vertical resolution than MIPAS frequently observe layered structures of different PSC types. Table 2 summarizes the $p_{i, j}$ values for all 13 classification areas of the current version (V1.2.8) of the classifier. Note that the probabilities $p_{i, j}$ were chosen empirically. The correlation diagrams of the modelled CSDB spectra (e.g. Figs. 4 and 6) provide guidance for a realistic parameter choice. The sensitivity of the classification result to the choice of the probabilities $p_{i, j}$ is presented in Sect. 3.4.

Figure 9 shows the PDF of the normalized PSC type probabilities for all cloudy spectra in the altitude range 12 to $30 \mathrm{~km}$ for SH June 2009. The PDFs for ice, NAT, and STS illustrate the well-defined separation of ice and non-ice events by the large count numbers for $P_{\text {ice }}>85 \%$, even higher for $P_{\text {ice }}<5 \%$, and very low values between the two extremes. For STS and NAT a significant number of the cloud observations show $P$ values between 20 and $50 \%$, indicating some difficulties to separate both types from each other. However, both types also show a significant number of spectra with large $P$ values $\left(P_{i}>65 \%\right)$ and suggest a robust separation by the new classification approach in the MIPAS measurements.

\subsection{Sensitivity test of the probability matrix}

We applied a Monte Carlo (MC) approach to infer the sensitivity of the classification results with respect to changes 

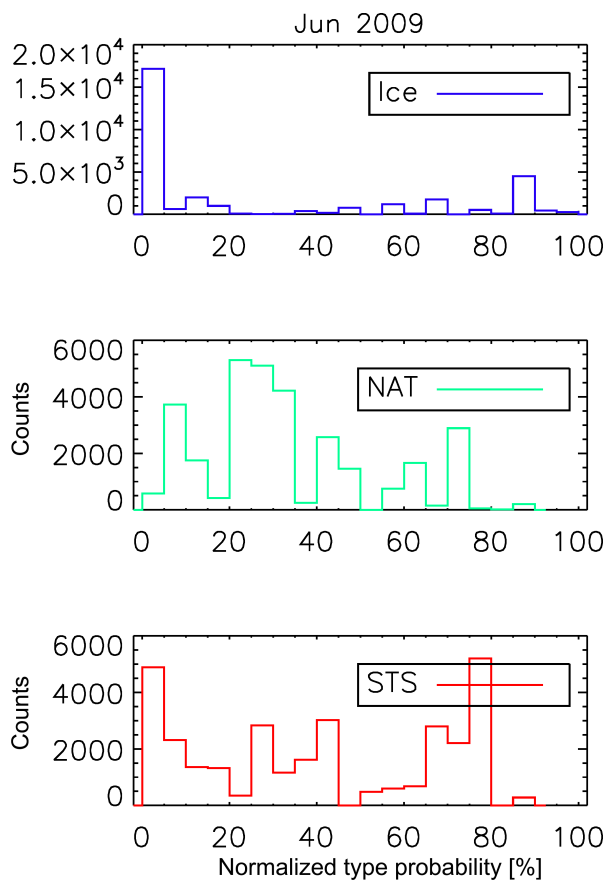

Figure 9. Event counts of the normalized probabilities $P_{j}$ for ice, NAT and STS $_{\text {mix }}$ of the Bayesian classifier for SH June 2009 cloudy spectra above $12 \mathrm{~km}$ altitude.

in the predefined probability matrix $p_{i, j}$ in Table 2 . Gaussian perturbations with a standard deviation of 5 percentage points were applied to the original $p_{i, j}$ values. The classification was repeated 2000 times for the same input dataset of real MIPAS spectra with the perturbed probability matrix. In the example for the SH in June 2011 shown in Fig. 10 nearly 18000 PSC spectra are analysed. We selected June of the MC test for two reasons. Firstly, June is the month when usually all three PSC types exist in the SH (Pitts et al., 2009; Di Liberto et al., 2014). Secondly, in the SH during June the appearance of each cloud type is characterized by a step-like process, whereby first STS, then NAT, and finally ice PSC form. This helps to minimize uncertainties caused by patchy distributions of mixed-type clouds in midwinter and late winter. However, the results for other months and years are similar.

The original result of the classification approach (undisturbed probability matrix) shown in Fig. 10 suggests a rather balanced occurrence of the three PSC types (25-30\%) and only a small amount of the NAT_STS mixed-type class $(\sim 6 \%)$. Additionally $5 \%$ of the analysed spectra are characterized as "unknown", indicating that the product probabilities for ice, NAT, and STS are all smaller than $40 \%$ for each class. The mean of the MC results shows a similar result for the three main classes and the unknown class, which give confidence that the selected probability matrix provides a robust classification. The definition of the probability matrix of version 1.2.8 (Table 2) has a higher tendency to select the

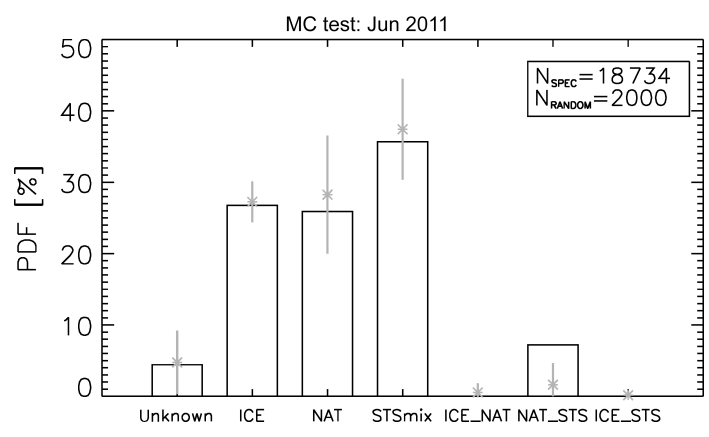

Figure 10. Probability distribution of the Bayesian classifier applied to the MIPAS spectra in the SH polar region in June 2011. Superimposed in grey are the mean value (stars) and standard deviation (error bars) of a Monte Carlo simulation varying the PSC type probability matrix (see text for details). The analysis considers only spectra at tangent heights between 16 and $28 \mathrm{~km}$ and up to $6 \mathrm{~km}$ below the CTH.

mixed-type STS_NAT compared to the results suggested by the MC approach. However, the final choice of $p_{i, j}$ follows the intention of a more conservative classification approach that accounts for possible uncertainties of the differentiation between NAT and STS.

\subsection{Comparison of 2CR and Bayesian classifier}

Various studies in the scientific literature applied the 2CR method for PSC classification in IR limb measurements (e.g. Spang et al., 2003, 2005; Höpfner et al., 2006b, 2009; Eckermann et al., 2009; Lambert et al., 2012; Arnone et al., 2012). Its main strength is the classification of clouds where the radiances are dominated by NAT particles with radii smaller than $3 \mu \mathrm{m}$ (Höpfner et al., 2006a). However, Spang et al. (2012) already showed that ice particles can overlap the STS region, and vice versa; STS with large VDP may overlap the region attributed to ice (Fig. 3).

Figure 11 presents a comparison of $2 \mathrm{CR}$ with our new combined Bayesian classifier for the three cloud types (ice, NAT, STS). MIPAS spectra are analysed on a monthly basis between 2007 and 2011 for the NH and SH potential PSC seasons (November-March for the NH and May-October for the SH; SH PSC periods are highlighted by the grey shaded sectors). Obviously, for months with strong ice formation potential (for SH the July-September period), the ice detection is significantly increased in the Bayes approach. In some years an enhancement even up to $30 \%$ can be observed and sums up to total amount for ice to up to $50 \%$ of all classified spectra (August 2011). The incorporation of additional wavelength regions sensitive to ice emissions seems to improve the capability to classify ice spectra substantially. Furthermore, the partitioning between STS and NAT also changes with the new approach. This becomes obvious for the $\mathrm{NH}$ PSC occurrences, where ice typically only plays a minor role in total abundance of PSCs due to the significantly warmer 


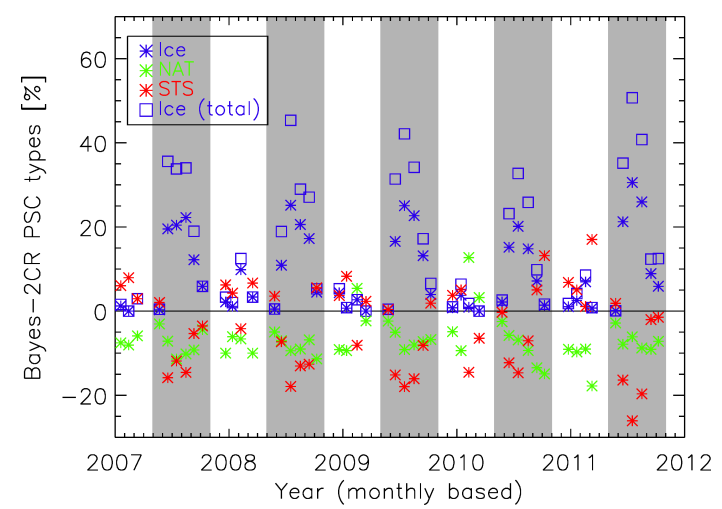

Figure 11. Differences in partitioning of the three PSC types between the new Bayesian classifier and the 2CR method based on all classified MIPAS spectra for each month of the years. SH analyses are highlighted by the grey shaded areas; $\mathrm{NH}$ data are in the white areas. In addition, the probability of the Bayes ice class with respect to all analysed PSC spectra is superimposed (blue open squares). This number visualizes the relevance of ice clouds at the specific time of the PSC season. For the comparison, we added the Bayes STS_NAT class to the STS class, which is in line with the definition of three classes attributed by the $2 \mathrm{CR}$ method (for details see text).

vortex temperatures. Here, the Bayesian classifier identifies more STS in contrast to NAT, but for specific winter conditions (e.g. 2009/10) this tendency is reversed. Overall, the changes are significantly smaller than for the ice class.

The general large abundance of the ice class compared to NAT and STS for both methods in the SH winters might be related to sampling effects. The numbers overestimate the partitioning of ice with respect to the other classes due to two rationales: (1) the satellite sampling results in a specifically high measurement density at the highest latitudes $(|\phi|=80$ $90^{\circ}$ ), where in the $\mathrm{SH}$ midwinter the coldest temperatures occur and the statistics suggest that ice is the most likely PSC type (up 50\%). (2) Spectra at tangent heights below optical thick clouds are usually also flagged as cloudy and will most likely be classified as the overlying cloud because the emissions are dominated by the optically thick spectrum in the layer above. The respective spectrum may be even cloudfree or of a different cloud type, but is masked by the cloud layer above. The cloud scenarios of the CSDB show that optically thick spectra $(\mathrm{CI}<1.3)$ are most likely to be observed for PSDs of ice clouds. Due to the large water abundance (3-5 ppmv) compared to the limited $\mathrm{HNO}_{3}$ abundance (3-15 ppbv) in the stratosphere, volume densities of ice can reach significantly larger values than NAT and STS. As a result of item (1) and (2), the number of ice clouds can easily be overestimated by limb measurements, especially when the atmospheric conditions favour the frequent formation of optically thick ice clouds, which is typically expected for $\mathrm{SH}$ midwinter conditions (July/August).

\section{Data analysis}

We applied a Bayesian classifier to the full MIPAS dataset from July 2002 to March 2012. The period includes a couple of longer data gaps caused by technical problems with the interferometer (e.g. Fischer et al., 2008), with the main gap between April and December 2004. However, the final dataset still provides excellent geographical and temporal coverage for $10 \mathrm{NH}$ and $9 \mathrm{SH}$ polar winters of daytime and night-time observations. This gives the opportunity for detailed case studies of polar processes related to PSC formation as well as for the compilation of a unique pole-covering climatology of PSC types. Further information on access to these data can be found in Sect. 6 .

\subsection{A case study of gravity-wave-induced PSC formation}

Höpfner et al. (2006b) showed the importance of mountain waves over the Antarctic Peninsula for the formation of an Antarctic NAT belt that was observed for the first time by MIPAS in the SH winter 2003. Currently, scientific interest is growing for a better assessment of the importance of gravity waves on the formation process of PSCs and a better representation of these processes in CCMs for more precise ozone predictions (Alexander et al., 2013; Orr et al., 2015). The new dataset of observations of PSC types for multiple $\mathrm{SH}$ and $\mathrm{NH}$ winter conditions up to pole may help to better constrain and validate new microphysical models describing the homogenous and heterogeneous formation processes for ice and NAT (e.g. Engel et al., 2013; Hoyle et al., 2013). These models are beyond the status of simple equilibrium schemes, which are still used in most CTMs and CCMs. A first study of using the new NAT scheme in the global chemical transport models CLaMS shows promising results (Grooß et al., 2014).

Figure 12 presents an example of the daily evolution of the PSC distribution of the Bayesian classifier for June 2010. The coloured symbols represent the PSC classes, where in addition to the three main classes (ice, STS, NAT), the three mixed types, unspecified, and optically thick clouds are also highlighted. Underlaid gridded $15 \mu \mathrm{m}$ brightness temperature variances retrieved from AIRS are presented as a proxy for the gravity wave (GW) activity in the altitude range around 20-25 km (see Sect. 2). It is obvious that the first outbreak of orographic GWs on 1 June has no imprint on the formation of NAT clouds in the outer region of the polar vortex. Wind conditions and temperature development of the cold pole do not favour the formation of PSCs by temperature variations induced by this mountain wave event. In contrast, the June 11 event immediately creates signals for NAT clouds in the downstream region of the Antarctic Peninsula. Until around 22 June large areas of the polar vortex in the temperature regime well above $T_{\text {ice }}$ are filled with cloud events attributed to NAT. Most of these NAT events seem to origi- 

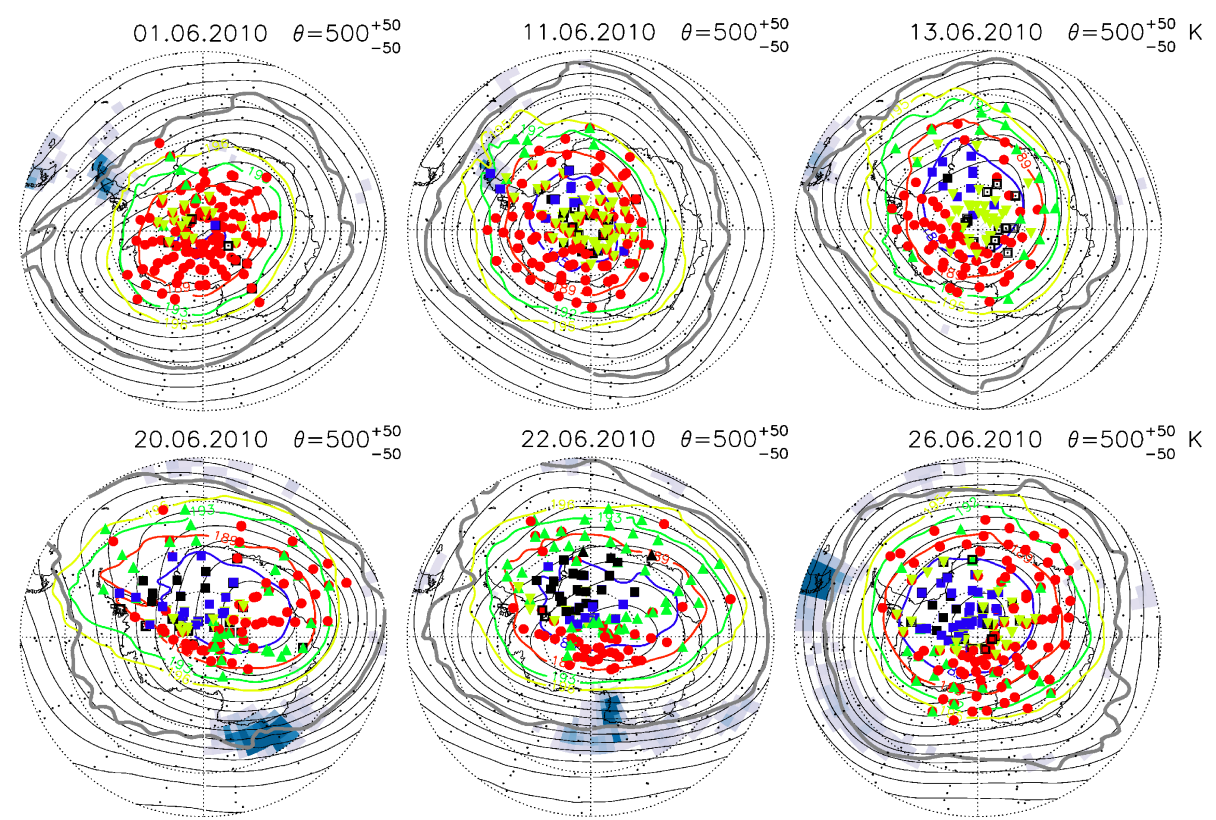

$$
\begin{aligned}
& \text { 口: Unspec. } \\
& \text { घ: ICE } \\
& \text { А: NAT } \\
& \bullet: \text { STSmix }
\end{aligned}
$$

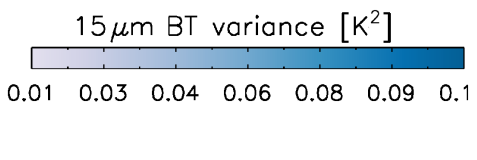

$$
\begin{aligned}
& \bullet: \text { ICE_NAT } \\
& \nabla: \text { NAT_STS } \\
& \text { : ICE_STS } \\
& \text { ஐ: Thick }(\mathrm{Cl}<1.3)
\end{aligned}
$$

Figure 12. Coloured symbols represent daily PSC distribution of the Bayesian classifier for 1, 11, 13, 20, 22, and 26 June 2011 in the potential temperature altitude range $500 \mathrm{~K} \pm 50 \mathrm{~K}$ equivalent to $\sim 20 \mathrm{~km}$ geometric altitude. Black dots highlight the MIPAS profile location. Superimposed in colour-coded grid boxes with $4^{\circ}$ longitude $\times 2^{\circ}$ latitude resolution in the background show brightness temperature variances from AIRS as a proxy for GW activity in the lower stratosphere. Black contour lines represent the Montgomery stream function computed from ERA Interim (Dee et al., 2011) data and illustrate the large-scale geostrophic flow conditions. The potential vorticity-based vortex boundary is highlighted by a dark grey contour. In addition colour-coded temperature contours represent the existing threshold temperatures $T_{\mathrm{NAT}}+2 \mathrm{~K}, T_{\mathrm{NAT}}, T_{\mathrm{STS}}$ (estimated by $\left(T_{\mathrm{NAT}}+T_{\mathrm{ice}}\right) / 2$ ), and $T_{\text {ice }}$ in yellow, green, red, and blue for constant stratospheric values for $\mathrm{HNO}_{3}$ (9 ppbv) and $\mathrm{H}_{2} \mathrm{O}$ (4 ppmv) and according to the formula by Hanson and Mauersberger (1988) and Marti and Mauersberger (1993).

nate from transport processes along the superimposed Montgomery stream function. These contours roughly represent the streamlines of the geostrophic wind and the NAT clouds are along contours crossing the AP region. These observations are in line with the mountain wave seeding hypothesis for PSCs as described and proofed with AIRS and MIPAS data by Eckermann et al. (2010) or to the MIPAS NAT-belt events in mid-June 2003 and 2008 previously reported by Höpfner et al. (2006b) and Lambert et al. (2012), respectively. Preliminary analyses of all MIPAS and AIRS SH observations suggest that generally, the early winter conditions in mid-June facilitate meteorological conditions to form a mountain-wave-induced NAT belt (8 out of 10 winter). However, we also found similar sporadic outbreaks in the region of the Antarctic Peninsula in a couple of September observations. These usually do not develop to the full extent of a NAT belt, where a nearly complete ring of NAT clouds fills the outer region of the cold pole region inside the vortex. This case study demonstrates that the BC provides a physically reasonable classification in a well-defined meteorological setting.

\subsection{Overall behaviour of the new classification approach}

The new PSC type classification approach for MIPAS provides data for the entire time of the Envisat mission (June 2002-March 2012). With the restriction to tangent altitudes between 15 and $35 \mathrm{~km}$, north and south of 55 and $-55^{\circ}$ latitude respectively, we found more than $14000 \mathrm{PSC}$ profiles out of 205000 profiles in the $\mathrm{NH}$ and more than 108000 profiles out of 255000 in the SH winter season. The PSC seasons are defined from November to March and from May to October, respectively.

Figure 13 summarizes the classification into the main groups of cloud types (ice, NAT, STS, NAT_STS, and unknown) for Arctic and Antarctic winter conditions with respect to all potential PSC spectra of MIPAS. The total number of PSC spectra and the partitioning between the type classes highlight the general difference in the meteorological conditions between the northern and southern polar vortices. The much colder and prolonged cold pool region in the Antarctic favours a much more intensive vortex-wide PSC 


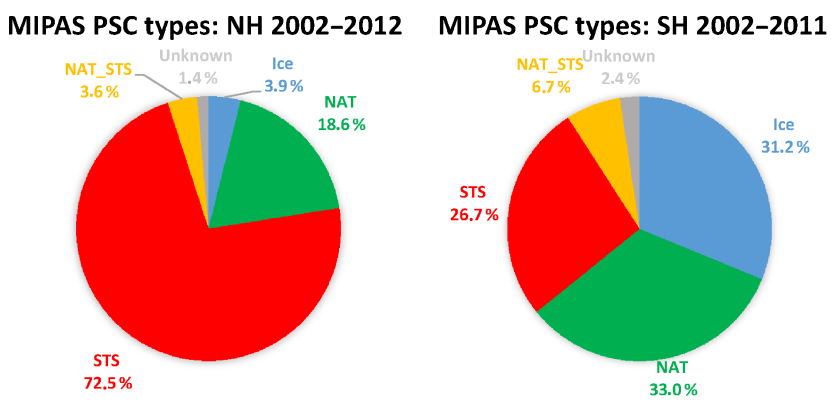

Figure 13. Breakdown of PSC types observed by MIPAS in the Arctic (December-March, left) and Antarctic (May-October, right) from 2002 to 2012. The percentages are averages over 10 and 9 seasons respectively, and relate to the total number of analysed spectra north/south of $55 /-55^{\circ}$ latitude. Spectra more than $6 \mathrm{~km}$ below the actual CTH in the profile are not taken into account. In total 44622 cloudy spectra are analysed for the NH and 317699 for SH.

formation and significantly longer PSC season than in the Arctic. There, the temperatures are usually not low enough to form larger areas with ice PSCs. Consequently, the percentage of ice (4\%) is significantly smaller than NAT (19\%), and STS $(72 \%)$ is the dominating cloud type in the $\mathrm{NH}$ winter. In contrast, the SH shows par-like conditions between the ice, NAT, and STS (31, 33, and $27 \%)$.

In comparison to the statistics of Pitts et al. (2011) for four winter of CALIOP measurements (2006-2010) in the SH, MIPAS shows significantly larger ice abundance (31\%) than CALIOP (12\%, Fig. 3 in Pitts et al., 2011), whereas in the $\mathrm{NH}$, both instruments show a very similar partitioning of 3.9 and $3.2 \%$, respectively. The large SH difference is only partly a surprising fact and not necessarily related to potential differences in detection sensitivity for specific PSC and mixed-PSC types between the different measurement techniques. Most of these differences can be attributed to the different latitudinal coverage of both instruments. MIPAS has the advantage of covering the complete polar cap, whereas CALIOP is restricted to a maximum latitude of $82^{\circ}$. Overlapping orbits at high latitudes increase the measurement density in particular for MIPAS. Together with the fact that the SH cold pool for most winters is centred exactly over the South Pole, the large abundance of ice clouds' observations in SH for MIPAS can be explained by differences in the sampling and orbit geometry between MIPAS and CALIOP to a large extent. As a test, we restricted the MIPAS observations to a maximum latitude of $80^{\circ} \mathrm{N} / \mathrm{S}$ and applied the classification to the same winters of the CALIOP statistics. The result shows a reduction for the ice partition from 31 to $20 \%$. However, the remaining value is still a significantly larger ice fraction than in the CALIOP analysis (12\%) and indicates more fundamental limitations and difficulties for comparisons between CALIOP and MIPAS. A more detailed discussion of these limitations is presented in the next section.

\subsection{MIPAS-CALIOP coincidence comparison}

For the verification of the MIPAS PSC classification, only ground-based and space-borne lidar PSC classification are available. Due to the fundamentally different measurement geometries affecting vertical and horizontal resolution as well as measurement sensitivity, the potential for meaningful comparisons is limited. Since CALIOP is the only other instrument with similar global and temporal coverage to MIPAS, we decided to only compare MIPAS with CALIOP.

The high vertical and along-track resolution and a wellestablished classification scheme (Pitts et al., 2009) make the CALIOP dataset valuable for PSC research (e.g. Peter and Grooß, 2013; WMO, 2014). The good temporal overlap of the CALIOP dataset with the MIPAS measurements (Jul 2007-Mar 2012) allows a detailed comparison with the new classification approach. The high measurement frequency for both satellites and similar coverage results in a high statistical significance for a comparison in spite of quite stringent coincidence criteria.

\subsubsection{Principles and sensitivities of nadir-limb comparisons}

The extremely highly resolved vertical and along-track measurements of CALIOP result in a kind of hyper-sampling of PSC structures compared to the MIPAS observations. MIPAS has to cope with the so-called limb path smearing effect (Spang et al., 2012, 2015). Figure 14 illustrates this effect, for an example where MIPAS and CALIOP measure in the same orbit plane. Such a constellation is regularly achieved in the A-Train constellation for several of the NASA Earth observation satellites and instruments (e.g. CALIPSO and MLS), but is not the situation for the MIPAS and CALIOP orbits (similar inclination but different orbit latitudes and equator crossing times). For better illustration of a typical coincidence, Fig. 14 shows the along-track CALIOP PSC type observations overlaid with the MIPAS line of sights of several limb scans along the CALIOP orbit track, where we assume that both satellites operate in the same orbit plane. Usually a coincidence is characterized by a crossing point of both orbits (profile with coloured squares for the MIPAS classification result). The next and the previous MIPAS profiles (thin lines) are already outside of the defined match radius $(\Delta x=100 \mathrm{~km})$. The long limb path through the atmosphere causes the limb smearing effect, where the information on the start and endpoint of the cloud along the line of sight is not retrievable from a single limb scan. The detection of a cloudy spectrum is attributed to the original tangent height, although the entrance in the cloud along the line of sight can be located in front of or behind the tangent point. This results in an uncertainty for the retrieved CTH. Spectra with tangent heights below the CTH might falsely be indicated as cloudy due to the cloud layer above (e.g. Höpfner et al., 2009). In contrast, the long limb path is a great advantage for the ob- 


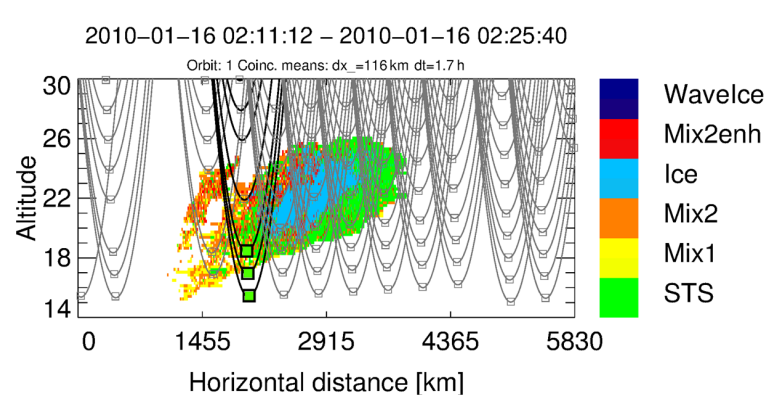

Figure 14. Visualization of the MIPAS limb paths (black lines) and a coincident MIPAS profile (coloured black-framed squares; other profiles do not fulfil the coincidence criteria) compared to the CALIOP high-resolution nadir measurements of PSC types (coloured pixels). The colour bar refers to the CALIOP PSC type classification. For details see text.

servation of optically very thin clouds and aerosols because the measurement integrates the signal along the line of sight.

Spang et al. (2015) showed that an ADP $>10^{7} \mu \mathrm{m}^{2} \mathrm{~cm}^{-2}$ is detectable for such as MIPAS (assuming a completely filled vertical and cross-track FOV). This threshold $\left(\mathrm{ADP}_{\text {thres }}\right)$ is equivalent to an ice water path of $0.3 \mathrm{~g} \mathrm{~m}^{-2}$, which corresponds to a cloud layer with $100 \mathrm{~km}$ and $1 \mathrm{~km}$ horizontal extent to an IWC of 0.003 and $0.3 \mathrm{mg} \mathrm{m}^{-3}$, respectively. These small water concentrations illustrate the extreme sensitivity of the IR limb cloud detection, even if the IWC is concentrated in a cloud that only partially fills the FOV. For the estimates, we assumed a cloud filling the complete cross-track and vertical FOV and a constant effective radius ( $R_{\text {eff }}$ ) of $10 \mu \mathrm{m}$. A thinner cloud layer will almost linearly increase the detection threshold, depending on where the cloud layer is placed in the FOV of MIPAS (Spang et al., 2015).

We investigated potential differences in the detection sensitivity for both instruments in more detail. For the standard archived V1-00 CALIOP PSC mask, the estimated minimum detectable STS volume density is $0.25 \mu \mathrm{m}^{3} \mathrm{~cm}^{-3}$ (L. Poole, personal communication, 2015). This value is applicable at $180 \mathrm{~m}$ vertical $\times 135 \mathrm{~km}$ horizontal resolution, the coarsest mean values applied in the dataset to improve the signal-tonoise ratio. The MIPAS detection sensitivity for STS is a volume density of the order of $0.45 \mu \mathrm{m}^{3} \mathrm{~cm}^{-3}$ and is based on the simulated spectra of the CSDB and the compact correlation between CI and $\log (\mathrm{VDP})$, with applied threshold values of $\mathrm{CI}_{\text {thres }}=4.5$ in the detection algorithm and the identical horizontal integration length through the cloud. Consequently, both instruments show a similar detection sensitivity. However, not all coincidences for the CALIOP measurements have such a long horizontal extent of $135 \mathrm{~km}$, and observations of the same cloud structures by both instruments may not have the same horizontal extent in the individual measurement due to the different orbit geometry (see above).
The MIPAS detection threshold is independent on any averaging due to the inertial integrating measurement technique in contrast to the CALIOP product. This allows a better detection sensitivity than CALIOP under certain conditions, for example for optically thin clouds close to and below the threshold value of CALIOP with a longer horizontal extent along the MIPAS line of sight than the maximum $135 \mathrm{~km}$ averaging of the CALIOP data processing.

\subsubsection{Coincidence statistics}

For the coincidence comparisons we applied very stringent miss-time $(\Delta t=2 \mathrm{~h})$ and miss-distance $(\Delta x=100 \mathrm{~km})$ criteria. This implies observation geometries where one MIPAS profile is coincident with an orbit segment of hundreds of individual CALIOP measurements (Fig. 14). For example, a maximum horizontal extent of $200 \mathrm{~km}(2 \times \Delta x)$ combined with the vertical FOV of MIPAS of $3 \mathrm{~km}$ results in a maximum number of CALIOP observations (pixels) of $(200 \mathrm{~km} / 5 \mathrm{~km}) \times(3 \mathrm{~km} / 0.18 \mathrm{~km})=680$ pixels. Instead of analysing these large numbers of coincident CALIOP pixels separately, we combined the whole set of CALIOP pixels and compare the most likely PSC type with the corresponding MIPAS observation. This MIPAS-like CALIOP PSC type is estimated by assigning the CALIOP type with the maximum count number in the ensemble. A mean CALIOP coincidence is only attributed as "no-cloud" (non-cloudy) if each coincident CALIOP pixel shows no cloud indication at all. In addition, we took care about the MIPAS difficulties to quantify the vertical extent of the cloud below the $\mathrm{CTH}$ and restricted the analysis to up to two altitude steps below the CTH.

Vertical and horizontal smoothing of CALIOP measurements within the MIPAS measurement volumes would be another and more precise approach for the comparison of the observations. However, horizontal and vertical smoothing of the CALIOP cloud classification flags is not applicable to provide physically meaningful results. Instead, smoothing should be applied to the CALIOP radiance measurements (Level 1 data) and classification being applied subsequently. This would imply a complete reprocessing of the CALIOP dataset and the consolidation of the specific new product, which is technically not feasible. Our simplified method considers the vertical and horizontal smoothing of MIPAS in the comparison with the CALIOP high-resolution measurements to a certain extent, but may create caveats in the coincidence statistics. For instance, we found a bias in comparison of the ice class, which will be further discussed below.

Figure 15 summarizes the count statistics for each specific PSC class for CALIOP and for MIPAS with respect to the PSC classes of the coincident measurement of the corresponding instrument. We selected a $\mathrm{SH}$ winter mean of three successive winters for the comparison, which results already in excellent count statistics of more than 12000 MIPAS profiles with coincident CALIOP information. 

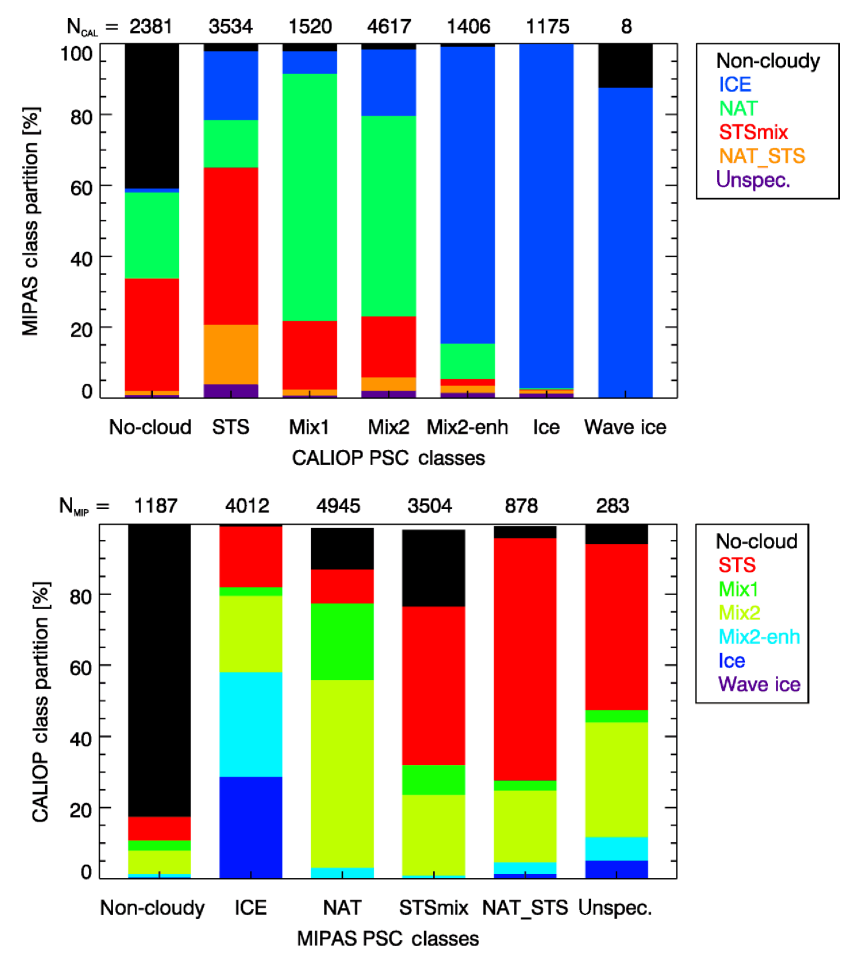

Figure 15. The MIPAS-CALIOP coincidence count statistics of the mean SH winter seasons 2009-2011 (May-October). A miss time of $\Delta t<2 \mathrm{~h}$ and miss distance of $\Delta x<100 \mathrm{~km}$ is applied together with the estimation of an MIPAS-like mean CALIOP type, and only observations above $16 \mathrm{~km}$ are taken into account (for details see text). Top: partitioning of the coincident MIPAS classes for the coincident CALIOP classification results. Bottom: partitioning of the coincident CALIOP classification for the MIPAS classes. Numbers on top of the coloured bars indicate the number of observations of the corresponding PSC class.

Taking the CALIOP measurements as reference (Fig. 15, top) the ice classification of MIPAS (>97\%) seems to perfectly match with the CALIOP ice class. It is also promising that the small amount of CALIOP wave ice (Sect. 2.3) agrees very well with MIPAS ice (seven out of eight events). The Mix 2-enhanced class (most likely NAT clouds with high volume densities with a little probability for ice clouds, Sect. 2.3) is dominated by MIPAS ice coincidences ( $80 \%)$ with a certain fraction of NAT $(\sim 10 \%)$. CALIOP Mix 1 and Mix 2 classes are largely assigned to the MIPAS NAT class, with 70 and $56 \%$ respectively. For STS, CALIOP provides a very robust classification because STS is the only PSC type without depolarization. However, the MIPAS classifier distributes the partitioning nearly equally over the different classes, with $44 \%$ STS and $16 \%$ NAT_STS, whereas $40 \%$ were attributed to ice and NAT. For the 2381 coincidences where CALIOP shows no indications for clouds, MIPAS only detects non-cloudy conditions in $\sim 40 \%$ of the events as well, but in total more than $55 \%$ NAT and STS $_{\text {mix }}$ spectra. This part of the comparison suggests a higher detec-
Table 3. MIPAS-like PSC types partitioning for CALIOP with respect to ice fraction $\left(f_{\mathrm{ICE}}\right) ; f_{\mathrm{ICE}}=0.5$ represents a fraction $>50 \%$; type contribution is given in $\%$.

\begin{tabular}{rrrrrrrr}
\hline$f_{\text {ICE }}$ & no-cloud & STS & Mix 1 & Mix 2 & Ice & Mix 2-enh & Wave-ice \\
\hline 0.50 & 0.7 & 17.1 & 2.4 & 21.5 & 28.4 & 29.4 & 0.2 \\
0.30 & 0.7 & 16.7 & 2.4 & 21.5 & 32.3 & 25.9 & 0.3 \\
0.10 & 0.7 & 12.5 & 2.3 & 19.4 & 50.7 & 13.0 & 1.0 \\
0.05 & 0.7 & 10.6 & 2.2 & 17.7 & 59.1 & 7.6 & 1.7 \\
0.01 & 0.6 & 6.1 & 2.0 & 12.4 & 74.0 & 1.4 & 3.0 \\
0.001 & 0.6 & 3.5 & 1.5 & 6.9 & 86.7 & 0.3 & 0.0 \\
\hline
\end{tabular}

tion sensitivity for MIPAS than for the CALIOP product; but the difference to CALIOP can also be an indication for a kind of hypersensitivity in the MIPAS cloud detection algorithm, for example if the method detects optically very thin background aerosol as PSC. The spectral dependence of sulfuric acid has many more similarities to NAT and STS compared to ice, and consequently, falsely detected aerosol should be most likely classified as NAT or STS (55\%) and not as ice $(<5 \%)$.

Taking the MIPAS classes as reference (Fig. 15, bottom), the comparison of the non-cloudy events shows better agreement than the CALIOP reference comparison (Fig. 15, top), with only $20 \%$ of the CALIOP coincidences classified as cloudy compared to $60 \%$ of MIPAS coincidences being classified as cloudy for no-cloud CALIOP conditions. For the other CALIOP classes (Fig. 15, bottom) the MIPAS ice class events only show a CALIOP ice class in $30 \%$ of the coincidences. The NAT class coincidences are distributed mainly to two CALIOP types, Mix $1(21 \%)$ and Mix $2(53 \%)$, and only minor contributions for STS $(<10 \%)$, Mix 2-enh $(<3 \%)$ and nearly no indication for ice $(0.1 \%)$. For the $\mathrm{STS}_{\text {mix }}$ class, $45 \%$ of the CALIOP coincidences are classified as STS, whereby only Mix 2 (may include larger number densities of NAT) of the CALIOP mixed-type classes contributes significantly $(23 \%)$. Here a relatively large part of $22 \%$ are nocloud events for CALIOP.

The impression of large differences in the ice classification between both instruments taken MIPAS as reference might be created by different sensitivities for ice, NAT, and STS of the two instruments. This causes difficulties in the case of observations of mixed-type clouds along the limb path. Due to the much bigger abundance of water vapour $(\sim 5 \mathrm{ppmv})$ in the stratosphere compared to nitric acid ( $5-15 \mathrm{ppbv})$ and consequently higher volume densities, ice clouds create significantly stronger signals in the primary measurement quantities of MIPAS and CALIOP (IR radiances and attenuated backscatter, respectively). Therefore, already a minor partition of CALIOP ice pixel in the MIPAS-like FOV box creates such a strong signal that these emissions can dominate the total radiances integrated along the line of sight and measured by MIPAS.

Table 3 investigates the effect of varying ice fraction ( $\left.f_{\mathrm{ICE}}\right)$ threshold in the MIPAS-like FOV mean information 
for the coincident CALIOP measurements. We applied a simple approach to test the sensitivity of the ice fraction for the MIPAS ice class coincidences by changing the threshold condition from the original $f_{\mathrm{ICE}}=0.5$ down to 0.001 . Obviously, this reduction has a very strong influence on the comparison of the MIPAS ice class with the CALIOP ice class. Reducing $f_{\text {ICE }}$ from 0.3 to 0.1 , the ice amount is increased to $\sim 50 \%$ by a reduction in the Mix 2-enh class (attributed to mixed-type of NAT and ice, Pitts et al., 2012). Finally, the ice partition increases up to $>85 \%$ for a reduction of $f_{\mathrm{ICE}}$ down to 0.001 at the expenses of the Mix 2 class. An ice fraction $f_{\text {ICE }}=0.001$ represents a minimum of just one cloudy ice pixel in the $3 \mathrm{~km} \times 200 \mathrm{~km}$ MIPAS-like FOV box. The sensitivity study indicates an overemphasis effect for ice in the observation of mixed-type clouds for the MIPAS Bayesian classifier, which needs consideration in the comparison. It seems difficult to quantify a representative threshold value precisely. In future studies, radiative transfer model calculations with different mixtures of PSC types could help to better estimate the threshold values.

\section{Summary}

We presented a new classification approach for different polar stratospheric clouds. The approach is developed for IR limb measurements by the MIPAS instruments on board the ESA Envisat satellite. The so-called Bayesian classifier combines the information content of various correlation diagrams of colour ratios and brightness temperature differences in the wavenumber region $790-1450 \mathrm{~cm}^{-1}$, covering several atmospheric window regions. The classifier estimates the most likely probability that one of the three PSC types (ice, NAT, or STS) dominates the spectral characteristics in a MIPAS spectrum affected by clouds. In addition, mixed-type clouds are defined, where none of the three type probabilities is $>50 \%$ but two are in the intermediate range of $40-50 \%$.

In a first comparison with coincident CALIOP measurements, excellent count statistics were achieved over several PSC seasons even though we applied restrictive miss-time and miss-distance criteria. Overall, the comparison shows good consistency between both instruments, even though the PSC classes are based on different measurement quantities, and the effect of mixed-type clouds can be very different for each instrument. The latter fact explains differences in the classification of mixed-type clouds in part. Especially the complementary viewing geometries (nadir/limb), whereby MIPAS always integrates over a large horizontal distance and CALIOP represents a more hyper-sampled pixel measurement, create some general caveats for a comparison of cloud types retrieved from both types of instrument.

The entire MIPAS measurement period from July 2002 to April 2013 is processed with the Bayesian classifier and constitutes a unique dataset of daytime and night-time PSC measurements up to the poles. Climatological mean winter statistics for PSC type occurrence frequencies and height resolved statistics of PSC area of the polar vortex over all winters can now be analysed. The dataset has the potential to be used for the validation of current chemical transport models with sophisticated microphysical schemes, or to improve climate chemistry models with more simple heterogeneous chemistry modules. In this way, the dataset can potentially help to improve the predictability of the future polar stratospheric ozone trends by these models.

In a case study for the SH polar vortex using MIPAS PSC data, together with AIRS analyses on the gravity wave activity over Antarctica, we showed the potential for the synergetic use of various remote sensing instruments to explore mountain-wave-induced PSC formation. This capability will be investigated in more depth over multiple years in an ongoing study by combining AIRS and MIPAS data together with results from the UK Unified Model, including a parameterization for sub-grid mountain wave processes (Orr et al., 2015). This may help to improve global models with respect to this only rarely considered effect in CCM and CTMs.

\section{Data availability}

Interested scientists can obtain access to the complete data record of the MIPAS PSC classification (version 1.2.8) by contacting the leading author (r.spang @fz-juelich.de). For the near future it is planned that the dataset be made publicly available together with CALIOP and in situ PSC data under the umbrella of a new activity in Stratosphere-troposphere Processes and their Role in Climate (SPARC, 2016).

Acknowledgements. The authors would like to thank ESA for providing MIPAS level $1 \mathrm{~b}$ data and funding of the MIPclouds study as well as NASA for providing CALIOP data. Reinhold Spang thanks Ines Tritscher (FZJ) for support with the visualization of the CALIOP data. Part of this work is inspired by discussions during the 1st PSC Initiative (PSCi) workshop funded by the International Space Science Institute (ISSI) in Bern, Switzerland.

The article processing charges for this open-access publication were covered by a Research Centre of the Helmholtz Association.

Edited by: C. von Savigny

Reviewed by: three anonymous referees

\section{References}

Achtert, P. and Tesche, M.: Assessing lidar-based classification schemes for polar stratospheric clouds based on 16 years of measurements at Esrange, Sweden, J. Geophys. Res., 119, 13861405, doi:10.1002/2013JD020355, 2014.

Alexander, S. P., Klekociuk, A. R., McDonald, A. J., and Pitts, M. C.: Quantifying the role of orographic gravity 
waves on polar stratospheric cloud occurrence in the Antarctic and the Arctic, J. Geophys. Res.-Atmos., 118, 11493-11507, doi:10.1002/2013JD020122, 2013.

Arnone, E., Castelli, E., Papandrea, E., Carlotti, M., and Dinelli, B. M.: Extreme ozone depletion in the 2010-2011 Arctic winter stratosphere as observed by MIPAS/ENVISAT using a 2D tomographic approach, Atmos. Chem. Phys., 12, 9149-9165, doi:10.5194/acp-12-9149-2012, 2012.

Aumann, H. H., Chahine, M. T., Gautier, C., Goldberg, M. D., Kalnay, E., McMillin, L. M., Revercomb, H., Rosenkranz, P. W., Smith, W. L., Staelin, D. H., Strow, L. L., and Susskind, J.: AIRS/AMSU/HSB on the Aqua Mission: Design, Science Objective, Data Products, and Processing Systems, IEEE T. Geosci. Remote Sens., 41, 253-264, 2003.

Avery, M., Winker, D., Heymsfield, A., Vaughan, M., Young, S., $\mathrm{Hu}$, Y., and Trepte, C.: Cloud ice water content retrieved from the CALIOP space-based lidar, Geophys. Res. Lett., 19, L05808, doi:10.1029/2011GL050545, 2012.

Baran, A. J.: On the scattering and absorption properties of cirrus cloud, J. Quant. Spectrosc. Ra., 89, 17-36, doi:10.1016/j.jqsrt.2004.05.008, 2004.

Biermann, U. M., Luo, B. P., and Peter, T.: Absorption spectra and optical constants of binary and ternary solutions of $\mathrm{H}_{2} \mathrm{SO}_{4}$, $\mathrm{HNO}_{3}$, and $\mathrm{H}_{2} \mathrm{O}$ in the mid infrared at atmospheric temperatures, J. Phys. Chem., 104, 783-793, 2000.

CALIPSO Science Team: CALIPSO/CALIOP Level 2, Polar Stratospheric Cloud Data, version 1.00, Hampton, VA, USA, NASA Atmospheric Science Data Center (ASDC), available at: https://eosweb.larc.nasa.gov/project/calipso/cal_lid_12_ pscmask-prov-v1-00_table, last access: July, 2015.

Carslaw, K. S., Luo, B. P., and Peter, T.: An analytical expression for the composition of aqueous $\mathrm{HNO}_{3}-\mathrm{H}_{2} \mathrm{SO}_{4}-\mathrm{H}_{2} \mathrm{O}$ stratospheric aerosols including gas phase removal of $\mathrm{HNO}_{3}$, Geophys. Res. Lett., 22, 1877-1880, doi:10.1029/95GL01668, 1995.

Carslaw, K. S., Wirth, M., Tsias, A., Luo, B. P., Dörnbrack, A., Leutbecher, M., Volkert, H., Renger, W., Bacmeister, J. T., Reimer, E., and Peter, T.: Increased stratospheric ozone depletion due to mountain-induced atmospheric waves, Nature, 391, 675-678, doi:10.1038/35589, 1998.

Chahine, M. T., Pagano, T. S., Aumann, H. H., Atlas, R., Barnet, C., Blaisdell, J., Chen, L., Divakarla, M., Fetzer, E. J., Goldberg, M., Gautier, C., Granger, S., Hannon, S., Irion, F. W., Kakar, R., Kalnay, E., Lambrigtsen, B. H., Lee, S., Marshall, J. L., McMillan, W. W., McMillin, L., Olsen, E. T., Revercomb, H., Rosenkranz, P., Smith, W. L., Staelin, D., Strow, L. L., Susskind, J., Tobin, D., Wolf, W., and Zhou, L.: AIRS: improving weather forecasting and providing new data on greenhouse gases, B. Am. Meteorol. Soc., 87, 911-926, 2006.

Clarisse, L., Coheur, P.-F., Prata, F., Hadji-Lazaro, J., Hurtmans, D., and Clerbaux, C.: A unified approach to infrared aerosol remote sensing and type specification, Atmos. Chem. Phys., 13, 21952221, doi:10.5194/acp-13-2195-2013, 2013.

Dee, D. P., Uppala, S. M., Simmons, A. J., Berrisford, P., Poli, P., Kobayashi, S., Andrae, U., Balmaseda, M. A., Balsamo, G., Bauer, P., Bechtold, P., Beljaars, A. C. M., van de Berg, L., Bidlot, J., Bormann, N., Delsol, C., Dragani, R., Fuentes, M., Geer, A. J., Haimberger, L., Healy, S. B., Hersbach, H., Holm, E. V., Isaksen, L., Kallberg, P., Koehler, M., Matricardi, M., McNally, A. P., Monge-Sanz, B. M., Morcrette, J. J., Park, B. K., Peubey,
C., de Rosnay, P., Tavolato, C., Thepaut, J. N., and Vitart, F.: The ERA-Interim reanalysis: configuration and performance of the data assimilation system, Q. J. Roy. Meteor. Soc., 137, 553-597, doi:10.1002/qj.828, 2011.

Di Liberto, L., Cairo, F., Fierli, F., Di Donfrancesco, G., Viterbini, M., Deshler, T., and Snels, M.: Observation of polar stratospheric clouds over McMurdo (77.85 $\left.{ }^{\circ} \mathrm{S}, 166.67^{\circ} \mathrm{E}\right)$ (2006-2010), J. Geophys. Res.-Atmos., 119, doi:10.1002/2013JD019892, 2014.

Drdla, K. and Müller, R.: Temperature thresholds for chlorine activation and ozone loss in the polar stratosphere, Ann. Geophys., 30, 1055-1073, doi:10.5194/angeo-30-1055-2012, 2012.

Eckermann, S. D., Hoffmann, L., Höpfner, M., Wu, D. L., and Alexander, M. J.: Antarctic NAT PSC belt of June 2003: Observational validation of the mountain wave seeding hypothesis, Geophys. Res. Lett., 36, L02807, doi:10.1029/2008GL036629, 2009.

Engel, I., Luo, B. P., Pitts, M. C., Poole, L. R., Hoyle, C. R., Grooß, J.-U., Dörnbrack, A., and Peter, T.: Heterogeneous formation of polar stratospheric clouds - Part 2: Nucleation of ice on synoptic scales, Atmos. Chem. Phys., 13, 10769-10785, doi:10.5194/acp13-10769-2013, 2013.

Eyring, V., Arblaster, J. M., Cionni, I., Sedlácek, J., Perlwitz, J., Young, P. J., Bekki, S., Bergmann, D., Cameron-Smith, P., Collins, W. J., Faluvegi, G., Gottschaldt, K. D., Horowitz, L. W., Kinnison, D. E., Lamarque, J. F., Marsh, D. R., SaintMartin, D., Shindell, D. T., Sudo, K., Szopa, S., and Watanabe, S.: Long-term ozone changes and associated climate impacts in CMIP5 simulations, J. Geophys. Res. Atmos., 118, 5029-5060, doi:10.1002/jgrd.50316, 2013.

Fahey, D. W., Gao, R. S., Carslaw, K. S., Kettleborough, J., Popp, P. J., Northway, M. J., Holecek, J. C., Ciciora, S. C., McLaughlin, R. J., Baumgardner, D. G., Gandrud, B., Wennberg, P. O., Dhaniyala, S., McKinney, K., Peter, T., Salawitch, R. J., Bui, T. P., Elkins, J. W., Webster, C. R., Atlas, E. L., Jost, H., Wilson, J. C., Herman, R. L., and Kleinbohl, A: The detection of large $\mathrm{HNO}_{3}$-containing particles in the winter Arctic stratosphere, Science, 291, 1026-1031, doi:10.1126/science.1057265, 2001.

Fischer, H., Birk, M., Blom, C., Carli, B., Carlotti, M., von Clarmann, T., Delbouille, L., Dudhia, A., Ehhalt, D., Endemann, M., Flaud, J. M., Gessner, R., Kleinert, A., Koopman, R., Langen, J., López-Puertas, M., Mosner, P., Nett, H., Oelhaf, H., Perron, G., Remedios, J., Ridolfi, M., Stiller, G., and Zander, R.: MIPAS: an instrument for atmospheric and climate research, Atmos. Chem. Phys., 8, 2151-2188, doi:10.5194/acp-8-2151-2008, 2008.

Gerber, E. P. and Son, S.-W.: Quantifying the summertime Austral jet stream and Hadley Cell response to stratospheric ozone and greenhouse gases, J. Clim., 27, 5538-5559, 2014.

Grainger, R. G., Peters, D. M., Thomas, G. E., Smith, A. J. A., Siddans, R., Carboni, E., and Dudhia, A.: Measuring Volcanic Plume and Ash Properties from Space, in: Remote-sensing of Volcanoes and Volcanic Processes: Integrating Observation and Modelling, edited by: Pyle, D., Mather, T., and Biggs, J., The Geological Society Special Publication 380, doi:10.1144/SP380.7, 2013.

Griessbach, S., Hoffmann, L., Spang, R., and Riese, M.: Volcanic ash detection with infrared limb sounding: MIPAS observations and radiative transfer simulations, Atmos. Meas. Tech., 7, 14871507, doi:10.5194/amt-7-1487-2014, 2014.

Grooß, J.-U., Engel, I., Borrmann, S., Frey, W., Günther, G., Hoyle, C. R., Kivi, R., Luo, B. P., Molleker, S., Peter, T., Pitts, M. 
C., Schlager, H., Stiller, G., Vömel, H., Walker, K. A., and Müller, R.: Nitric acid trihydrate nucleation and denitrification in the Arctic stratosphere, Atmos. Chem. Phys., 14, 1055-1073, doi:10.5194/acp-14-1055-2014, 2014.

Grossmann, K. U., Offermann, D., Gusev, O., Oberheide, J., Riese, M., and Spang, R.: The CRISTA-2 mission, J. Geophys. Res., 107, 8173, doi:10.1029/2001JD000667, 2002.

Hanson, D. and Mauersberger, K.: Solubility and equilibrium vapour pressures of $\mathrm{HCl}$ dissolved in polar stratospheric cloud materials - Ice and the trihydrate of nitric acid, Geophys. Res. Lett., 15, 1507-1510, doi:10.1029/GL015i013p01507, 1988.

Höpfner, M.: Study on the impact of polar stratospheric clouds on high resolution mid-IR limb emission spectra, J. Quant. Spectrosc. Ra., 83, 93-107, 2004.

Höpfner, M., Oelhaf, H., Wetzel, G., Friedl-Vallon, F., Kleinert, A., Lengel, A., Maucher, G., Nordmeyer, H., Glatthor, N., Stiller, G. P., von Clarmann, T., Fischer, H., Kröger, C., and Deshler, T.: Evidence of scattering of tropospheric radiation by PSCs in mid-IR limb emission spectra: MIPAS-B observations and KOPRA simulations, Geophys. Res. Lett., 29, 119-1-119-4, doi:10.1029/2001GL014443, 2002.

Höpfner, M., Luo, B. P., Massoli, P., Cairo, F., Spang, R., Snels, M., Di Donfrancesco, G., Stiller, G., von Clarmann, T., Fischer, H., and Biermann, U.: Spectroscopic evidence for NAT, STS, and ice in MIPAS infrared limb emission measurements of polar stratospheric clouds, Atmos. Chem. Phys., 6, 1201-1219, doi:10.5194/acp-6-1201-2006, 2006a.

Höpfner, M., Larsen, N., Spang, R., Luo, B. P., Ma, J., Svendsen, S. H., Eckermann, S. D., Knudsen, B., Massoli, P., Cairo, F., Stiller, G., v. Clarmann, T., and Fischer, H.: MIPAS detects Antarctic stratospheric belt of NAT PSCs caused by mountain waves, Atmos. Chem. Phys., 6, 1221-1230, doi:10.5194/acp-6-1221-2006, $2006 \mathrm{~b}$

Höpfner, M., Pitts, M. C., and Poole, L. R.: Comparison between CALIPSO and MIPAS observations of polar stratospheric clouds, J. Geophys. Res., 114, D00H05, doi:10.1029/2009JD012114, 2009.

Hoffmann, L., Xue, X., and Alexander, M. J.: A global view of stratospheric gravity wave hotspots located with Atmospheric Infrared Sounder observations, J. Geophys. Res., 118, 416-434, doi:10.1029/2012JD018658, 2013.

Hoffmann, L., Alexander, M. J., Clerbaux, C., Grimsdell, A. W., Meyer, C. I., Rößler, T., and Tournier, B.: Intercomparison of stratospheric gravity wave observations with AIRS and IASI, Atmos. Meas. Tech., 7, 4517-4537, doi:10.5194/amt-7-4517-2014, 2014.

Hollstein, A., Fischer, J., Carbajal Henken, C., and Preusker, R.: Bayesian cloud detection for MERIS, AATSR, and their combination, Atmos. Meas. Tech., 8, 1757-1771, doi:10.5194/amt-81757-2015, 2015.

Hoyle, C. R., Engel, I., Luo, B. P., Pitts, M. C., Poole, L. R., Grooß, J.-U., and Peter, T.: Heterogeneous formation of polar stratospheric clouds - Part 1: Nucleation of nitric acid trihydrate (NAT), Atmos. Chem. Phys., 13, 9577-9595, doi:10.5194/acp13-9577-2013, 2013.

Hurley, J., Dudhia, A., and Grainger, R. G.: Retrieval of macrophysical cloud parameters from MIPAS: algorithm description, Atmos. Meas. Tech., 4, 683-704, doi:10.5194/amt-4-683-2011, 2011.
Lambert, A., Santee, M. L., Wu, D. L., and Chae, J. H.: A-train CALIOP and MLS observations of early winter Antarctic polar stratospheric clouds and nitric acid in 2008, Atmos. Chem. Phys., 12, 2899-2931, doi:10.5194/acp-12-2899-2012, 2012.

Li, J., Menzel, W. P., Yang, Z. D., Frey, R. A., and Ackerman, S. A.: High-spatial-resolution surface and cloud-type classification from MODIS multispectral band measurements, J. Appl. Meteorol., 42, 204-226, 2003.

Manney, G. L., Santee, M. L., Rex, M., Livesey, N. J., Pitts, M. C., Veefkind, P., Nash, E. R., Wohltmann, I., Lehmann, R., Froidevaux, L., Poole, L. R., Schoeberl, M. R., Haffner, D. P., Davies, J., Dorokhov, V., Gernandt, H., Johnson, B., Kivi, R., Kyrö, E., Larsen, N., Levelt, P. F., Makshtas, A., McElroy, C. T., Nakajima, H., Parrondo, M. C., Tarasick, D. W., von der Gathen, P., Walker, K. A., and Zinoviev, N. S.: Unprecedented Arctic ozone loss in 2011, Nature, 478, 469-475, doi:10.1038/nature10556, 2011.

Marti, J. and Mauersberger, K.: Laboratory simulations of PSC particle formation, Geophys. Res. Lett., 20, 359-362, doi:10.1029/93GL00083, 1993.

Mie, G.: Beiträge zur Optik trüber Medien, speziell kolloidaler Metallösungen, Ann. Phys.-Berlin, 330, 377-445, doi:10.1002/andp.19083300302, 1908.

Offermann, D., Grossmann, K. U., Barthol, P., Knieling, P., Riese, M., and Trant, R.: The CRyogenic Infrared Spectrometers and Telescopes for the Atmosphere (CRISTA) experiment and middle atmosphere variability, J. Geophys. Res., 104, 16311-16325, 1999.

Orr, A., Hosking, J. S., Hoffmann, L., Keeble, J., Dean, S. M., Roscoe, H. K., Abraham, N. L., Vosper, S., and Braesicke, P.: Inclusion of mountain-wave-induced cooling for the formation of PSCs over the Antarctic Peninsula in a chemistry-climate model, Atmos. Chem. Phys., 15, 1071-1086, doi:10.5194/acp-15-10712015, 2015.

Peter, T. and Grooß, J.-U.: Polar Stratospheric Clouds and Sulfate Aerosol Particles: Microphysics, Denitrification and Heterogeneous chemistry, in:Stratospheric Ozone Depletion and Climate Change, edited by: Müller, R., RSC Publishing, ISBN: 978-184973-002-0, p. 108-144, 2012.

Pitts, M. C., Poole, L. R., and Thomason, L. W.: CALIPSO polar stratospheric cloud observations: second-generation detection algorithm and composition discrimination, Atmos. Chem. Phys., 9, 7577-7589, doi:10.5194/acp-9-7577-2009, 2009.

Pitts, M. C., Poole, L. R., Dörnbrack, A., and Thomason, L. W.: The 2009-2010 Arctic polar stratospheric cloud season: a CALIPSO perspective, Atmos. Chem. Phys., 11, 2161-2177, doi:10.5194/acp-11-2161-2011, 2011.

Pitts, M. C., Poole, L. R., Lambert, A., and Thomason, L. W.: An assessment of CALIOP polar stratospheric cloud composition classification, Atmos. Chem. Phys., 13, 2975-2988, doi:10.5194/acp-13-2975-2013, 2013.

Polvani, L. M., Waugh, D. W., Correa, G. J. P., and Son S.-W.: Stratospheric Ozone Depletion: The Main Driver of Twentieth-Century Atmospheric Circulation Changes in the Southern Hemisphere, J. Climate, 24, 795-812, doi:10.1175/2010JCLI3772.1, 2011.

Raspollini, P., Carli, B., Carlotti, M., Ceccherini, S., Dehn, A., Dinelli, B. M., Dudhia, A., Flaud, J.-M., López-Puertas, M., Niro, F., Remedios, J. J., Ridolfi, M., Sembhi, H., Sgheri, L., and von Clarmann, T.: Ten years of MIPAS measurements with 
ESA Level 2 processor V6 - Part 1: Retrieval algorithm and diagnostics of the products, Atmos. Meas. Tech., 6, 2419-2439, doi:10.5194/amt-6-2419-2013, 2013.

Sembhi, H., Remedios, J., Trent, T., Moore, D. P., Spang, R., Massie, S., and Vernier, J.-P.: MIPAS detection of cloud and aerosol particle occurrence in the UTLS with comparison to HIRDLS and CALIOP, Atmos. Meas. Tech., 5, 2537-2553, doi:10.5194/amt-5-2537-2012, 2012.

Shibata, T., Iwasaka, Y., Fujiwara, M., Hayashi, M., Nagatani, M., Shiraishi, K., Adachi, H., Sakai, T., Susumu, K., and Nakura, Y.: Polar stratospheric clouds observed by lidar over Spitsbergen in the winter 1994/1995: Liquid particles and vertical "sandwich" structure, J. Geophys. Res., 102, 10829-10840, 1997.

Solomon, S.: Stratospheric ozone depletion: a review of concepts and history, Rev. Geophys., 37, 275-316, 1999.

Spang R. and Remedios, J., Observations of a distinctive infrared spectral feature in the atmospheric spectra of polar stratospheric clouds measured by the CRISTA instrument, Geophys. Res. Lett., 30, 1875-1878, doi:10.1029/2003GL017231, 2003.

Spang, R., Remedios, J. J., and Barkley, M., Colour Indices for the Detection and Differentiation of Cloud Types in Infra-red Limb Emission Spectra, Adv. Space Res., 33, 1041-1047, 2004.

Spang, R., Remedios, J. J., Kramer, L. J., Poole, L. R., Fromm, M. D., Müller, M., Baumgarten, G., and Konopka, P.: Polar stratospheric cloud observations by MIPAS on ENVISAT: detection method, validation and analysis of the northern hemisphere winter 2002/2003, Atmos. Chem. Phys., 5, 679-692, doi:10.5194/acp-5-679-2005, 2005a.

Spang, R., Remedios, J. J., Tilmes, S., and Riese, M., MIPAS observation of polar stratospheric clouds in the Arctic 2002/2003 and Antarctic 2003 winters, Adv. Space Res., 36, 868-878, 2005 b.

Spang, R., Griessbach, S., Höpfner, M., Dudhia, A., Hurley, J., Siddans, R., Waterfall, A., Remedios, J. J., and Sembhi., H.: Technical Note: Retrievability of MIPAS cloud parameter, ESA-ESRIN Contract No. 20601/07/I-OL, March, 2008.

Spang, R., Arndt, K., Dudhia, A., Griessbach, S., Höpfner, M., Hurley, J., Remedios, J. J., Sembhi, H., and Siddans, R.: Algorithm Technical Basis Document: Cloud Information Retrieval from MIPAS measurements, ESA-ESRIN Contract No. 20601/07/IOL, Version 2.1, 10 June, 2010a.

Spang, R., Höpfner, M., Dudhia, A., Siddans, R., Waterfall, A., Poulsen, C., Remedios, J. J., and Sembhi H.: Product Validation Report for the MIPAS cloud parameter processor, ESA-ESRIN Contract No. 20601/07/I-OL, 10 June, 2010b.

Spang, R., Arndt, K., Dudhia, A., Höpfner, M., Hoffmann, L., Hurley, J., Grainger, R. G., Griessbach, S., Poulsen, C., Remedios, J. J., Riese, M., Sembhi, H., Siddans, R., Waterfall, A., and Zehner, C.: Fast cloud parameter retrievals of MIPAS/Envisat, Atmos. Chem. Phys., 12, 7135-7164, doi:10.5194/acp-12-71352012, 2012.

Spang, R., Günther, G., Riese, M., Hoffmann, L., Müller, R., and Griessbach, S.: Satellite observations of cirrus clouds in the Northern Hemisphere lowermost stratosphere, Atmos. Chem. Phys., 15, 927-950, doi:10.5194/acp-15-927-2015, 2015.

SPARC: Stratosphere-troposphere Processes And their Role in Climate: Polar Stratospheric Cloud Activity, available at: http: //www.sparc-climate.org/activities/polar-stratospheric-clouds/, last access: 21 February, 2016.
Stiller, G. P. (Ed.): The Karlsruhe Optimized and Precise Radiative Transfer Algorithm (KOPRA), vol. FZKA 6487 of Wissenschaftliche Berichte, Forschungszentrum Karlsruhe, 2000.

Strawa, A. W., Drdla, K., Fromm, M., Pueschel, R. F. Hoppel, K. W., Browell, E. V., Hamill, P., and Dempsey, D. P.: Discriminating Types Ia and Ib polar stratospheric clouds in POAM satellite data, J. Geophys. Res., 107, 8291, doi:10.1029/2001JD000458, 2002.

Taylor, F., Lambert, A., Grainger, R., Rodgers, C., and Remedios, J.: Properties of Northern Hemisphere polar stratospheric clouds and volcanic aerosol in 1991/92 from UARS/ISAMS satellite measurements, J. Atmos. Sci., 51, 3019-3026, 1994.

Toon, O. B., Tolbert, M. A., Middlebrook, A. M., and Jordan, J.: Infrared optical constants of $\mathrm{H}_{2} \mathrm{O}$, ice, amorphous nitric acid solutions, and nitric acid hydrates, J. Geophys. Res., 99, 2563125654, 1994.

Weigel, R., Volk, C. M., Kandler, K., Hösen, E., Günther, G., Vogel, B., Grooß, J.-U., Khaykin, S., Belyaev, G. V., and Borrmann, S.: Enhancements of the refractory submicron aerosol fraction in the Arctic polar vortex: feature or exception?, Atmos. Chem. Phys., 14, 12319-12342, doi:10.5194/acp-14-12319-2014, 2014.

Wilks, D. S.: Statistical methods in the atmospheric sciences, 2nd. Edn., International Geophysics Series, Vol. 100, Academic Press, 2005.

Winker, D. M., Vaughan, M. A., Omar, A., Hu, Y., Powell, K. A., Liu, Z., Hunt, W. H., and Young, S. A. Overview of the CALIPSO Mission and CALIOP Data Processing Algorithms, J. Atmos. Ocean. Tech., 26, 2310-2323, doi:10.1175/2009JTECHA1281.1, 2009.

WMO (World Meteorological Organization): Scientific Assessment of Ozone Depletion 2014, World Meteorological Organization, Global Ozone Research and Monitoring Project-Report No. 55, Geneva, Switzerland, 416 pp., 2014.

Woiwode, W., Grooß, J.-U., Oelhaf, H., Molleker, S., Borrmann, S., Ebersoldt, A., Frey, W., Gulde, T., Khaykin, S., Maucher, G., Piesch, C., and Orphal, J.: Denitrification by large NAT particles: the impact of reduced settling velocities and hints on particle characteristics, Atmos. Chem. Phys., 14, 11525-11544, doi:10.5194/acp-14-11525-2014, 2014.

Woiwode, W., Höpfner, M., Bi, L., Pitts, M. C., Poole, L. R., Oelhaf, H., Molleker, S., Borrmann, S., Klingebiel, M., Belyaev, G., Ebersoldt, A., Griessbach, S., Grooß, J.-U., Gulde, T., Krämer, M., Maucher, G., Piesch, C., Rolf, C., Sartorius, C., Spang, R., and Orphal, J.: Spectroscopic evidence for large aspherical $\beta$-NAT particles involved in denitrification in the December 2011 Arctic stratosphere, Atmos. Chem. Phys. Discuss., doi:10.5194/acp-2016-146, in review, 2016.

Yang, P., Wei, H., Huang, H.-L. Baum, B. A., Hu, Y. X., Kattawar, G. W., Mishchenko, M. I., and Fu, Q.: Scattering and absorption property database for nonspherical ice particles in the nearthrough far-infrared spectral region, Appl. Opt. 44, 5512-5523, 2005.

Zasetsky, A. Y., Gilbert, K., Galkina, I., McLeod, S., and Sloan, J. J.: Properties of polar stratospheric clouds obtained by combined ACE-FTS and ACE-Imager extinction measurements, Atmos. Chem. Phys. Discuss., 7, 13271-13290, doi:10.5194/acpd7-13271-2007, 2007. 